\title{
A Novel Efficient Cluster-Based MLSE Equalizer for Satellite Communication Channels with M-QAM Signaling
}

\author{
Eleftherios Kofidis, ${ }^{1}$ Vassilis Dalakas, ${ }^{2}$ Yannis Kopsinis, ${ }^{3}$ and Sergios Theodoridis ${ }^{2}$ \\ ${ }^{1}$ Department of Statistics and Insurance Science, University of Piraeus, 80 Karaoli \& Dimitriou Street, 18534 Piraeus, Greece \\ ${ }^{2}$ Department of Informatics and Telecommunications, University of Athens, Panepistimioupolis, Ilissia, 15784 Athens, Greece \\ ${ }^{3}$ Institute for Digital Communications, School of Engineering and Electronics, the University of Edinburgh, Kings Buildings, \\ Mayfield Road, Edinburgh EH9 3JL, UK
}

Received 24 April 2005; Revised 19 December 2005; Accepted 18 February 2006

Recommended for Publication by Bernard Mulgrew

In satellites, nonlinear amplifiers used near saturation severely distort the transmitted signal and cause difficulties in its reception. Nevertheless, the nonlinearities introduced by memoryless bandpass amplifiers preserve the symmetries of the $M$-ary quadrature amplitude modulation ( $M$-QAM) constellation. In this paper, a cluster-based sequence equalizer (CBSE) that takes advantage of these symmetries is presented. The proposed equalizer exhibits enhanced performance compared to other techniques, including the conventional linear transversal equalizer, Volterra equalizers, and RBF network equalizers. Moreover, this gain in performance is obtained at a substantially lower computational cost.

Copyright () 2006 Hindawi Publishing Corporation. All rights reserved.

\section{INTRODUCTION}

The role of a satellite is to receive a signal from an earth station or another satellite (uplink) and, acting as a simple repeater, to transmit it to another earth station or satellite (downlink) [1]. The need to maximally exploit on-board resources in a satellite communication system often imposes driving a high power amplifier (HPA), such as the traveling wave tube amplifier (TWTA), at or near its saturation point, resulting in a nonlinear distortion of the signal, and rendering the overall link nonlinear. To overcome nonlinear distortions, constant modulus constellation symbols (e.g., 4QAM) are commonly used [2]. However, large QAM signal constellations have to be adopted whenever high bandwidth efficiency is required [3], resulting in severe nonlinear distortions. Two approaches have been proposed for solving the problem of correct reception of the transmitted signal in those cases: (a) equalization $[4,5]$ and (b) predistortion or power amplifier linearization [6-8].

Equalization refers to processing the signal at the receiver side in order to recover the transmitted data, thus postcanceling the link's nonlinear (amplifier) and linear (multipath) distortions. Conventional linear equalizers combat only the intersymbol interference (ISI), introduced by the propagation channel, while nonlinear equalizers aim also at equalizing the nonlinear effects of the HPA. The main drawback of the equalization approach is the additional cost and the computational load it entails for each terminal.

On the other hand, predistortion techniques aim at precanceling the nonlinear effects via modeling the inverse of the amplifier characteristic and predistorting the data prior to the amplification stage. The overall characteristic then becomes linear. The advantage of this approach lies in the fact that only a single system is needed for canceling the HPA nonlinearity at the satellite, compared to using an equalizer in each terminal. On the other hand, its main drawback is that the predistorter must be on-board, so it cannot be applied to the satellites already on orbit. Moreover, in case multipath is present, an equalizer at the terminal side is still needed.

In this paper, we will deal only with the first approach, namely, equalization at the receiver end. Relevant works commonly resort to nonlinear equalizers based on neural network (NN) structures [5, 9] or on Volterra series [10-12]. NN-based equalizers include multilayer perceptrons (MLP) $[13,14]$, radial basis functions (RBF) $[15,16]$, and self organizing maps (SOM) [17-19]. A comparative study of the performance of MLP, RBF, and SOM equalizers is given in [20]. However, NN and Volterra techniques, in addition to their high computational and implementation complexity, have the disadvantage of invariably requiring a large (often unrealistic) number of training samples to result in a satisfying solution $[5,11]$. Moreover, for high-order modulation 
formats (e.g., $M$-QAM, $M>4$ ), which are desirable for the purposes of spectral efficiency, reasonable results have only been obtained for low IBOs $^{1}$ [5] (i.e., near the linear region, e.g., $-6 \mathrm{~dB}$ IBO).

This work presents a novel method of equalizing satellite channels, which exhibits a very good performance for any rectangular ${ }^{2}$ QAM constellation, even at high IBOs (i.e., near saturation, $0 \mathrm{~dB} \mathrm{IBO}$ ). It is characterized by implementation simplicity, low computational cost, and the ability to provide a good solution with only a small number of training samples. This comes from an efficient exploitation of the symmetries underlying the modulation schemes, along with the special character of the AM/AM and AM/PM [21] nonlinearities in TWT amplifiers. The method is basically an adaptation to the satellite context of the so-called one-dimensional clusterbased sequence equalizer (1D CBSE), recently proposed for the linear channel case $[22,23]$. The latter is a maximum likelihood sequence estimation (MLSE) equalizer that circumvents the channel identification stage, which is required in standard MLSE equalizers. Instead, the points (centers) around which the noisy channel output samples are clustered are first estimated and then employed to calculate the path metrics needed in the Viterbi algorithm (VA). Moreover, the symmetries in the source constellation are exploited to dramatically reduce the number of cluster centers that need to be estimated directly from the training data, leading to substantial computational savings. The method has been shown to exhibit a very good (ML) performance, at a low computational complexity.

In this work, the extension of the 1D CBSE to memoryless TWT nonlinearities is considered. The idea of using MLSE, adopting Forney's approach with VA [24], in a bandlimited satellite channel was first presented in the mid-70's (see $[25,26]$ and the references therein) and until today a major concern in such methods is the processing complexity. The method to be presented here applies to regenerative payloads (used in new satellite generations, e.g., NASA's Advanced Communications Technology Satellite (ACTS) [27], or in the SkyPlex project [28]), where the transmitted symbols are made available on-board before the amplification $[7,8,29,30]$ via demodulation (therefore no account is taken here of the uplink channel and noise). ${ }^{3}$ The method exploits the nature of the TWT nonlinearity (dependence only on the modulus of the input signal) and appeals to the methodology of the 1D CBSE in order to provide a computationally cheap estimate of the cluster centers. Furthermore, the required training sequence is very short, compared to other previously used techniques (e.g., [31]).

In the following sections, we will describe in detail the proposed extended equalizer as well as experimental results from applying it with two rectangular $M$-QAM constellation

\footnotetext{
1 The amplifier input backoff (IBO) is defined as the ratio of the amplifier input signal power $\left(P_{\text {in }}\right)$ to the input saturation power $\left(P_{\text {sat }}\right)$ : IBO $(\mathrm{dB})=$ $10 \log _{10}\left(P_{\text {in }} / P_{\text {sat }}\right)$.

2 Cross QAM constellations $(M=8,32, \ldots)$ are not considered here.

3 The earth HPA introduces only a mild nonlinearity, hence the uplink channel can be considered as overall linear [26].
}

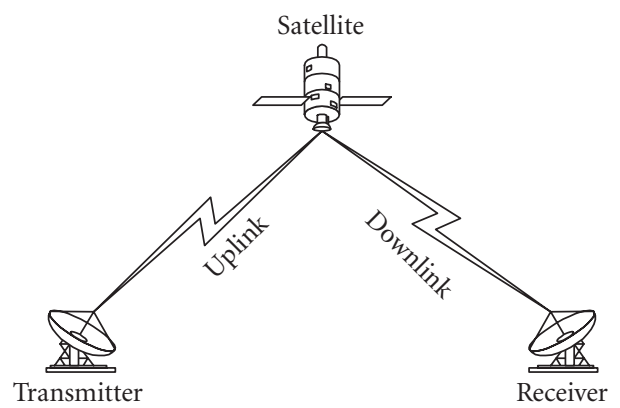

FIGURE 1: Satellite communication system.

schemes $(M=4,16)$ in an additive white Gaussian noise (AWGN) channel and a 2-tap stationary channel in downlink. The communication model is presented in Section 2. Section 3 provides a short review of the CBSE for the linear case. The preservation of $M$-QAM symmetries by memoryless nonlinear amplifiers is demonstrated in Section 4, where the new equalization method is presented. Experimental results along with computational complexities and performance comparisons with Volterra equalizers and RBF equalizers are presented in Section 5. Results of an LMS-running linear transversal equalizer are also given as a reference. Conclusions are drawn in Section 6.

\section{DESCRIPTION OF THE COMMUNICATION SYSTEM AND CHANNEL MODEL}

Figure 1 illustrates a typical satellite communication system [1]. Communication satellites have traditionally employed simple bent-pipe ${ }^{4}$ transponder relay designs. As mobile global communication systems are becoming more complex, new generation satellites have regenerative payloads $[2,28]$ with on-board processing. This means that the baseband transmitted signal is available on-board, via demodulation, and hence uplink and downlink can be treated separately. The proposed equalizer is to be applied to the downlink channel.

Figure 2(a) shows the downlink communication model. The digital signal to be transmitted is the data stream $u+j v$, assumed independent and identically distributed. The pulse shaping filter before the memoryless nonlinearity of the HPA is a square root raised cosine (SRRC) filter of sufficient bandwidth compared to the signal bandwidth. Therefore, ISI is introduced only by filters following the nonlinearity $[11,31]$. The adopted signaling scheme is the rectangular $M$-QAM. Figure 2(b) illustrates the baseband discrete equivalent communication system model for the downlink, where $x_{k}$ is the $k$ th transmitted symbol, which can take on one among $M$ distinct values from a source alphabet $S(S=\{a+j b \mid a, b=$ $(2 m-1-\sqrt{M}) \cdot d, m=1,2, \ldots, \sqrt{M}\}$ in $M$-QAM $), z_{k}$ is the

\footnotetext{
4 The simile with a "bent pipe" is often used for a transmission via a nonregenerative satellite transponder because the satellite simply retransmits the received signal back to the ground. That is, no symbol detection is involved.
} 


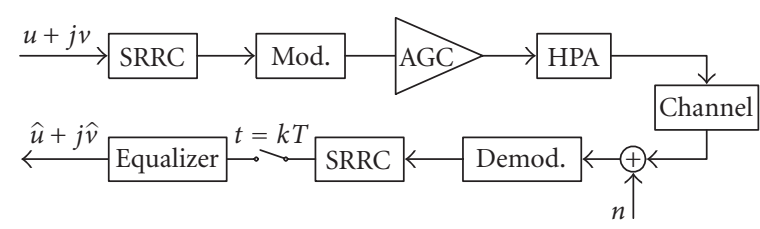

(a)

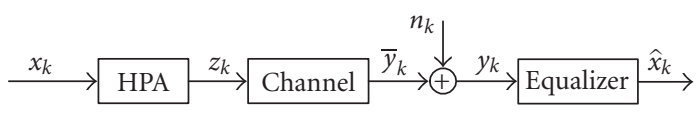

(b)

Figure 2: (a) The downlink communication system model and (b) its discrete equivalent.

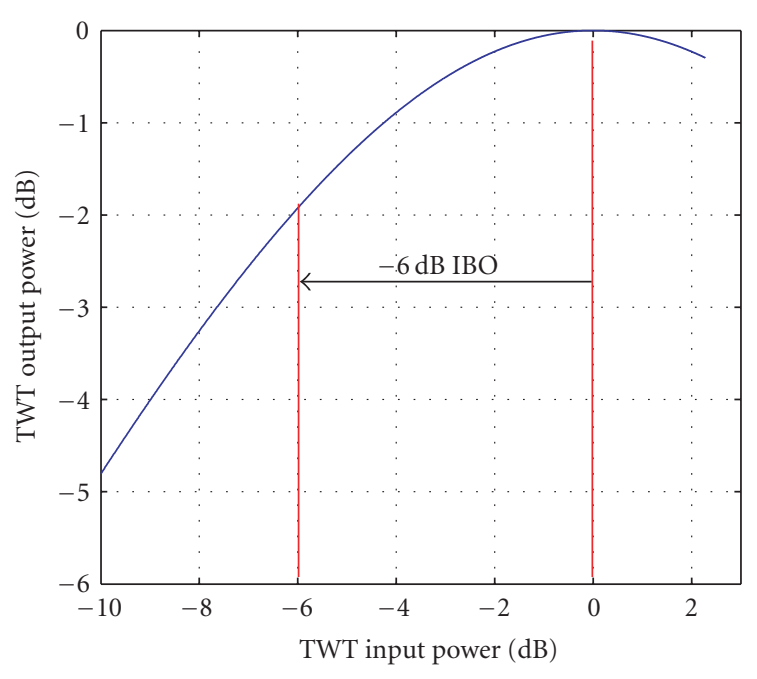

(a)

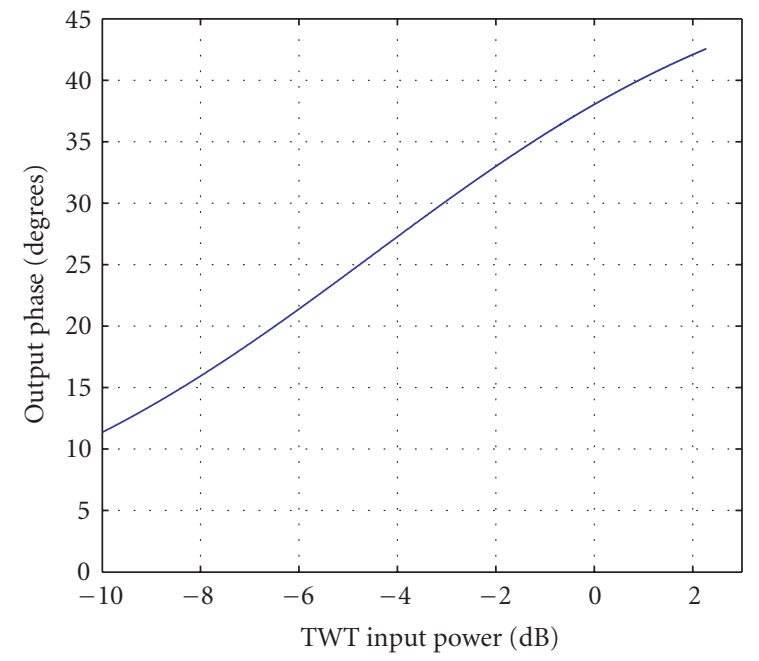

(b)

Figure 3: (a) AM/AM and (b) AM/PM conversions.

same symbol at the output of the nonlinear amplifier, $n_{k}$ is additive white Gaussian noise, uncorrelated with the channel input, $y_{k}$ denotes the $k$ th received observation, and $\hat{x}_{k}$ is the detected symbol.

There are two technologies for the high power amplifiers (HPA) on board satellites: traveling wave tube amplifiers (TWTA) and solid state power amplifiers (SSPA).

(i) TWTA can generally be considered as memoryless. They are characterized by an AM/AM conversion and an AM/PM conversion, as the ones illustrated in Figure 3 . These are commonly modeled by a Saleh model [21].

(ii) SSPA have intrinsic memory. It is common to model an SSPA with memory by a memoryless nonlinearity (see [32] for the type of the nonlinearity) followed by a linear IIR filter [6].

Here we will deal only with TWT amplifiers, ${ }^{5}$ due to their common use in satellites [33]. According to Saleh's model

\footnotetext{
${ }^{5}$ Nevertheless, it can be readily seen that the technique to be discussed here can also be applied in SSPA if the common approach of approximating the IIR filter following the memoryless nonlinearity with an FIR filter [6] is adopted. However the method can become prohibitively complex if the FIR filter has a large number of coefficients (60 in [6]).
}

[21], an input

$$
x(t)=A \cos \left(2 \pi f_{c} t+\theta\right)
$$

into a bandpass amplifier produces an output of the form $[34,35]$ :

$$
z(t)=g(A) \cos \left[2 \pi f_{c} t+\theta+\Phi(A)\right]
$$

where the nonlinear gain function $g(A)$ is commonly referred to as the $A M / A M$ characteristic and the nonlinear phase function $\Phi(A)$ is called the $A M / P M$ characteristic. These are expressed as

$$
\begin{gathered}
g(A)=\frac{\alpha_{a} A}{1+\beta_{a} A^{2}}, \\
\Phi(A)=\frac{\alpha_{p} A^{2}}{1+\beta_{p} A^{2}}
\end{gathered}
$$

and plotted in Figure 3, with parameters $\alpha_{a}, \beta_{a}, \alpha_{p}$, and $\beta_{p}$ assuming typical values from [21]. The common case of $-6 \mathrm{~dB}$ $\mathrm{IBO}$ is also shown. It is common practice to work with power back-off when nonconstant envelope modulation formats are used for transmission, although this implies less power efficiency. It will be shown that one of the advantages of our 
method is its ability to work with $M$-QAM $(M>4)$ constellation schemes, even at $0 \mathrm{~dB}$ IBO, where other methods fail.

The downlink communication channel after the TWT can be modeled as a finite impulse response (FIR) filter spanning over $L$ consecutive transmitted symbols, with transfer function $H(z)$. Thus, the received signal, sampled at the symbol transmission period, is given by

$$
y_{k}=\sum_{i=0}^{L-1} h_{i} z_{k-i}+n_{k}=\mathbf{h}^{T} \mathbf{z}_{k}+n_{k} \equiv \bar{y}_{k}+n_{k},
$$

where $^{6} \mathbf{h}=\left[h_{0}, h_{1}, \ldots, h_{L-1}\right]^{T}$ is the vector of the (generally complex) $L$ taps of the channel impulse response (CIR), $\mathbf{z}_{k}=\left[z_{k}, z_{k-1}, \ldots, z_{k-L+1}\right]^{T}$ is the vector of the transmitted symbols $\mathbf{x}_{k}=\left[x_{k}, x_{k-1}, \ldots, x_{k-L+1}\right]^{T}$ distorted by the memoryless nonlinearity, $\bar{y}_{k}$ denotes the noiseless observation associated with the above transmitted sequence of symbols, and $n_{k}$ is the additive white Gaussian noise, whose real and imaginary components are independent white sequences with equal variances, $\sigma^{2} / 2$, determined by the signal-to-noise ratio (SNR).

\section{THE 1D CLUSTER-BASED SEQUENCE EQUALIZER}

In this section, we will briefly review the 1D CBSE presented in [22], for linear channels, considering a channel model where the HPA part of our system (Figure 2) is omitted (linear case). The method proposed in [22] is an MLSE equalizer that circumvents the channel identification stage and exploits the symmetries in the source constellation along with the channel linearity to obtain ML performance at a reduced computational complexity.

Recall that the MLSE equalizer has first to compute an estimate, $\hat{\mathbf{h}}$, of the CIR, and then apply the VA (or one of its variants) to estimate the ML input sequence based on distances of the form ${ }^{7} D_{\mathbf{x}}=\left|y-\widehat{\mathbf{h}}^{T} \mathbf{x}\right|^{2}$. This entails a significant computational cost, since $M^{L}$ convolution sums $\widehat{\mathbf{h}}^{T} \mathbf{x}$ have to be computed per received sample, one for each of the $M^{L}$ combinations $\mathbf{x}$ of $L$ symbols from the alphabet $S$. The main idea in the 1D CBSE algorithm stems from the fact that it is the set of quantities $\bar{y}=\widehat{\mathbf{h}}^{T} \mathbf{x}$ that is needed in the VA and not the CIR itself; indeed, $D_{\mathbf{x}}=|y-\bar{y}|^{2}$. Moreover, these quantities are the noiseless channel outputs that coincide with the points (centers) around which the noisy observations are clustered due to the noise. Thus, they can be directly estimated via supervised clustering. The spread of the clusters depends on the power of the noise. The number of clusters as well as their position on the complex plane depend on the number and the values of the CIR taps.

Thus, the problem of explicit CIR estimation, as it is required by MLSE equalizers, can be circumvented and all that is needed is to estimate the $M^{L}$ centers $\bar{y}$ of the clusters formed on the complex plane. What is even more important

\footnotetext{
${ }^{6}$ Superscript ${ }^{T}$ denotes transposition.

7 Forney's scheme [24] is adopted here.
}

is that, by exploiting the constellation symmetry, direct (from the data) estimates for only $L$ appropriately chosen cluster centers suffice to yield the estimates for all $M^{L}$ of them.

To describe the estimation procedure, some definitions are first in order. The tap contribution, $c_{x}^{m}$, of the $m$ th tap, $h_{m}$, to the generation of a cluster center is the quantity

$$
c_{x}^{m}=x h_{m}
$$

with $x$ taking values from the symbol alphabet $S$. We can observe that $c_{x}^{m}$ can take one out of $M$ different values, depending on the value of the symbol $x$. For example, for $M=4$, we have the values $c_{1+j}^{m}, c_{-1+j}^{m}, c_{-1-j}^{m}$, and $c_{1-j}^{m}$. Using this notation, equation (5), for the received signal down to earth, can be rewritten as

$$
\bar{y}_{\left[x_{k}, x_{k-1}, \ldots, x_{k-L+1}\right]}=\sum_{m=0}^{L-1} c_{x_{k-m}}^{m},
$$

where $\bar{y}_{\left[x_{k}, x_{k-1}, \ldots, x_{k-L+1}\right]}$ denotes the cluster center associated with the transmitted $L$-tuple $\left[x_{k}, x_{k-1}, \ldots, x_{k-L+1}\right]$. Furthermore, it is easy to realize that, for each $h_{m}$, only one of the $M$ possible values, say $c_{x}^{m}$, needs to be estimated; all the rest can be obtained via multiplications as in $c_{x^{\prime}}^{m}=\left(x^{\prime} / x\right) \cdot c_{x}^{m}$. In the 4 -QAM case, this reduces to simple $\pi / 2$ rotations, for example, $c_{1-j}^{m}=-j c_{1+j}^{m}, c_{-1-j}^{m}=-c_{1+j}^{m}, c_{-1+j}^{m}=j c_{1+j}^{m}$.

The $L$ centers that have to be estimated directly from the observations can be chosen as follows. First, choose any of the $M^{L}$ centers, say $C_{\text {basic }}=\bar{y}_{\left[x_{0}, x_{1}, \ldots, x_{L-1}\right]}$, and call it $b a-$ sic center, and the associated $L$-tuple basic sequence, $\mathbf{x}_{\mathrm{basic}}=$ $\left[x_{0}, x_{1}, \ldots, x_{L-1}\right]$. Then the $L$ centers to be directly estimated from the data are those that correspond to the basic sequence with a sign change in one of its entries: $C_{0}=\bar{y}_{\left[-x_{0}, x_{1}, \ldots, x_{L-1}\right]}$, $C_{1}=\bar{y}_{\left[x_{0},-x_{1}, \ldots, x_{L-1}\right]}, \ldots, C_{L-1}=\bar{y}_{\left[x_{0}, x_{1}, \ldots,-x_{L-1}\right]}$.

A center $C_{m}$ can be estimated, for example, by averaging the associated observations, that is,

$$
C_{m}=\frac{1}{N^{(m)}} \sum_{k=1}^{N^{(m)}} y_{k}^{(m)}, \quad 0 \leq m \leq L-1,
$$

where $y_{k}^{(m)}$ is the $k$ th observation associated with $C_{m}$ and $N^{(m)}$ the number of these observations. The basic center $C_{\text {basic }}$ can be computed based on the estimates of the $L$ centers $C_{m}$ as follows [22]:

$$
C_{\text {basic }}=\frac{\sum_{m=0}^{L-1} C_{m}}{L-2}, \quad L>2 .
$$

Obviously, the above formula cannot be applied when $L \leq$ 2 . In such a case, $C_{\text {basic }}$ can be computed directly from the received observations as in (8).

The computation of the tap contributions for the symbols of the basic sequence is then straightforward:

$$
c_{x_{m}}^{m}=\frac{C_{\text {basic }}-C_{m}}{2}, \quad 0 \leq m \leq L-1 .
$$

From these $L$ estimated contributions (one for each tap) one can then easily compute the rest, $(M-1) L$, exploiting the 


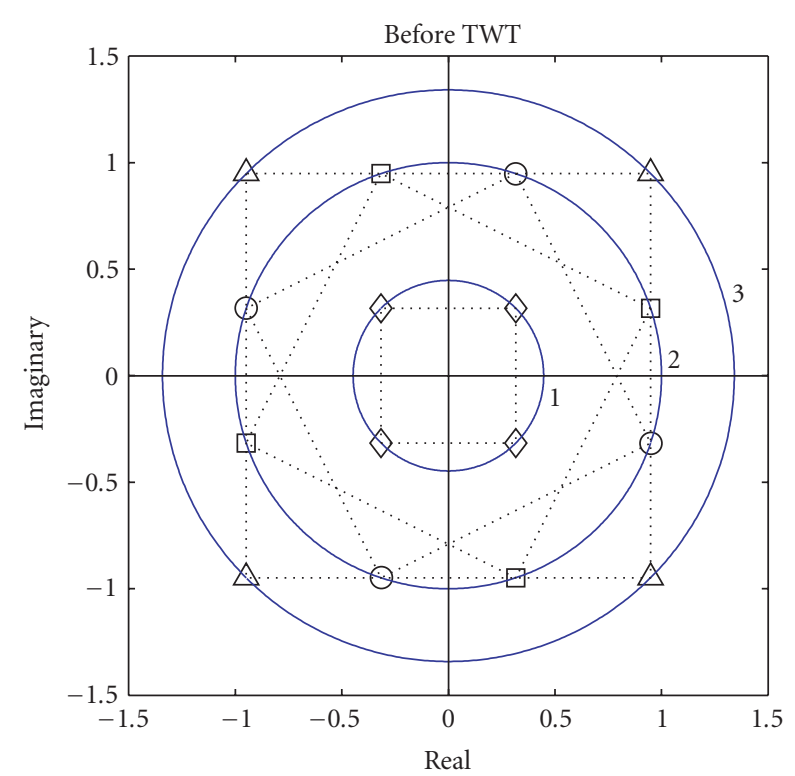

(a)

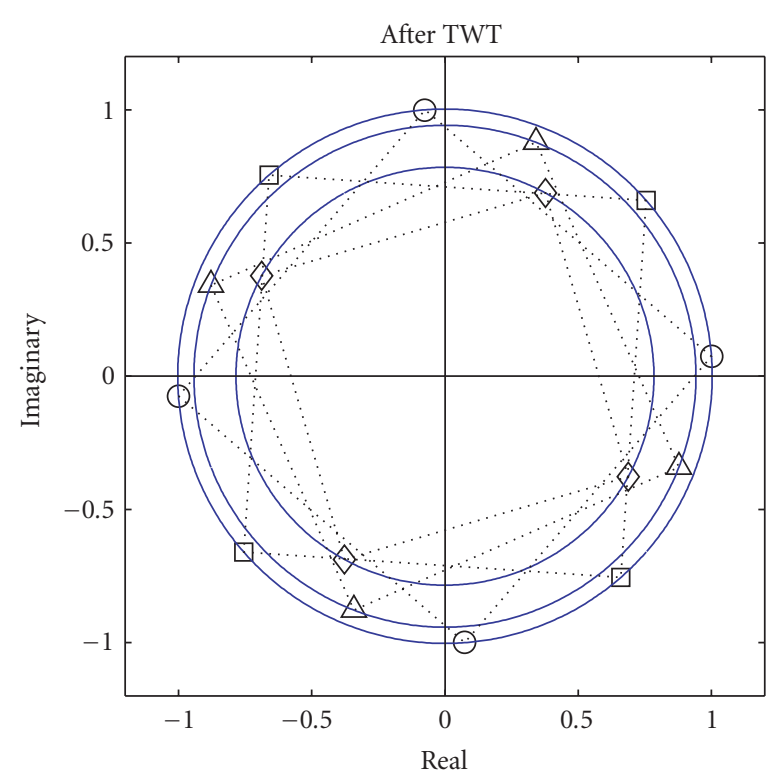

(b)

FIGURE 4: 16-QAM constellation at the (a) input and (b) output of the TWTA. The 3 energy levels and the 4 squares formed by the 16 constellation points are illustrated.

structure of the input constellation. Once all the tap contributions have been estimated, the remaining cluster centers are then computed as in (7).

If the training sequence that is employed to estimate the $L$ cluster centers $C_{m}, m=0,1, \ldots, L-1$, is to be as short and effective as possible, it has to "visit" these clusters as many times as possible and equally often. It turns out that, if only the input vectors corresponding to these $L$ centers are to appear in the training sequence, the symbols in the basic sequence should coincide, that is,

$$
x_{0}=x_{1}=\cdots=x_{L-1}=x .
$$

Such a training sequence can be constructed by periodically repeating the sequence $[\underbrace{x, x, \ldots, x}_{L-1 \text { times }},-x]$. For the case of $L=$ 2 , this has to be modified to $[x, x,-x]$, to include the basic sequence as well. ${ }^{8}$

\section{EXPLOITATION OF CONSTELLATION SYMMETRIES IN THE CASE OF MEMORYLESS NONLINEARITIES}

In this section, we will extend the above equalization method to the case where a TWTA (as in (3), (4)) is present. To this end, we will first need to clarify the way the nonlinearity affects the input constellation.

\footnotetext{
${ }^{8}$ In fact, this sequence visits the cluster for $[x,-x]$ twice as often as the cluster for $[x, x]$. One can do a little better than that if the sequence $[x, x, x,-x]$ is used instead, so that both clusters are represented equally often.
}

\subsection{Constellation symmetries}

The adopted signaling scheme, namely, rectangular $M$-ary QAM, may be viewed as a form of combined digital amplitude and digital phase modulation. In view of (1)-(4), the baseband complex envelope of the TWTA output is given by

$$
\begin{aligned}
\tilde{z}(t) & =g[A(t)] e^{j\{\theta(t)+\Phi[A(t)]\}} \\
& =\left[A(t) e^{j \theta(t)}\right]\left\{\frac{g[A(t)]}{A(t)} e^{j \Phi[A(t)]}\right\} \\
& \triangleq \tilde{x}(t) G(|\tilde{x}(t)|),
\end{aligned}
$$

where $\sim$ denotes complex envelope. In words, the output of the TWTA is the product of the input signal with a factor that depends only on the input amplitude. The result is an amplitude change and a phase rotation of the input signal constellation points. Equation (12) implies that the change is the same for all constellation points that share the same energy level. The $M$ symbols in the input constellation can be grouped in two possible ways (see Figure 4(a) for the example of 16-QAM):

(1) in $I$ circles on the complex plane, where $I$ is the number of the energy levels (for the 16-QAM case, $I=3$ ),

(2) in $M / 4$ squares (four points in each square) that are centered on the origin.

Observe that $M / 4$ points lie in each quadrant of the signal space. Since each of these $M / 4$ points is located the corner of one of the $M / 4$ squares, all $M$ points can result from such a group of $M / 4$ points via simple $n \cdot \pi / 2$ rotations, $1 \leq n \leq 3$. After the application of the (memoryless) nonlinearity, a new 


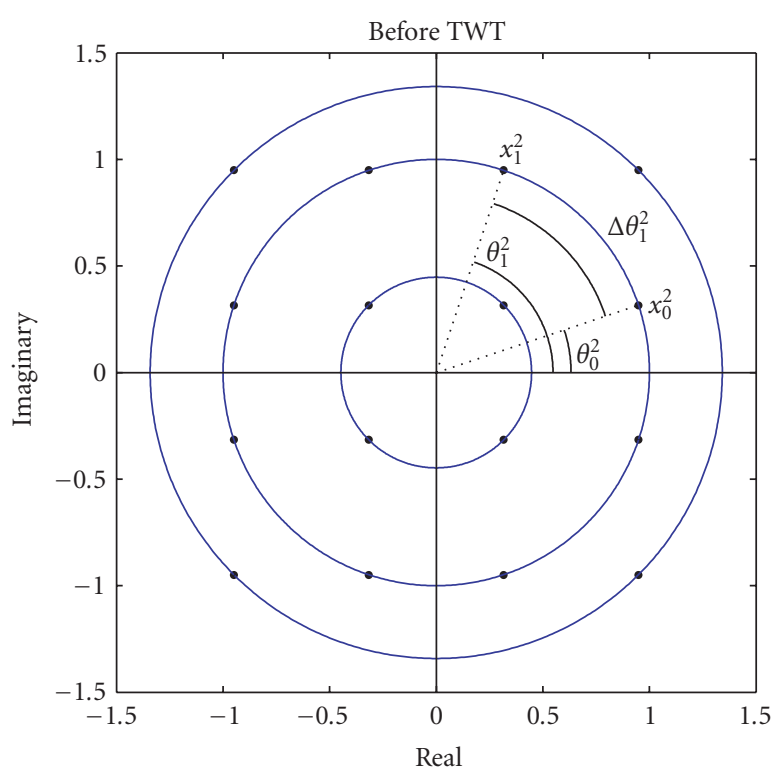

(a)

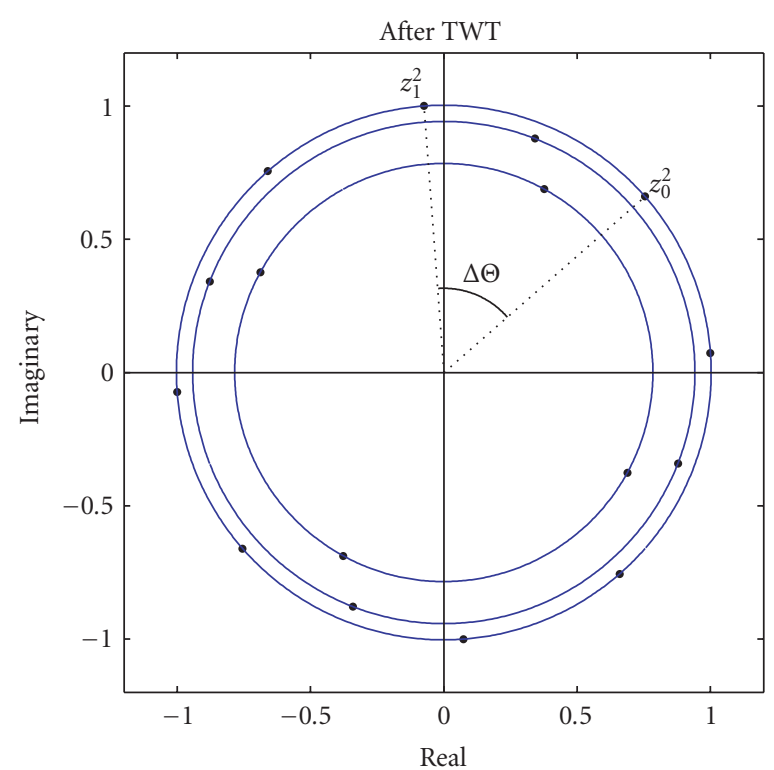

(b)

FIGURE 5: 16-QAM constellation at the (a) input and (b) output of the TWTA. Angles between equal modulus symbols are shown: $\Delta \Theta=\Delta \theta_{1}^{2}$.

constellation structure is formed. However, the number of the resulting points in the signal space is the same as before (Figure 4(b)). In Figure 4, corresponding points and energy levels have been drawn with the same type of lines, at the input, (a), and output, (b), of the TWTA. It is not difficult to see that the above symmetries $(1,2)$ of the constellation are preserved by the amplifier. This is a consequence of the fact that the angles between the constellation points that lie on the same energy circle remain unaltered (see Figure 5 and the appendix for a proof). Thus, the resulting points continue to form squares centered on the origin, as it was the case prior to the application of the nonlinearity. The length of the diagonal of each square is now equal to $2 \cdot g(A)$ and the angle of rotation, with respect to the corresponding square in the input constellation, is $\Phi(A)$, where $A$ is the amplitude of each of the four symbols on the corners of the square. Moreover, the number of energy levels is not affected by the TWTA, due to the nature of the nonlinearity. In the sequel, we will show how these symmetries can be efficiently exploited to reduce the total number of cluster centers to be estimated directly from the training sequence in the CBSE equalizer.

\subsection{Center estimation technique}

Assuming, as in Section 3, that a general $L$-taps linear filter, with impulse response $\mathbf{h}=\left[h_{0}, h_{1}, \ldots, h_{L-1}\right]^{T}$, follows the nonlinearity, we may redefine the tap contribution, $c_{x}^{m}$, of the $m$ th tap $h_{m}(6)$ to the generation of a cluster center to be the quantity

$$
c_{x}^{m}=z(x) h_{m},
$$

where $z(x)$ is the response of the TWTA to the input symbol $x$. We can observe that $c_{x}^{m}$ can take as many different values as the number of values of the symbol $x$. We show here that one needs to estimate, using the training data, only as many contribution values, for each channel tap, as the number $I$ of the different energy levels in the constellation. The rest can be obtained via rotations with fixed, a priori known angles. Once all the contributions have been computed, the estimates of all cluster centers become readily available via (7). These are then used in the VA.

As we have already seen, the $M$ points of the constellation are grouped in $M / 4$ squares and it suffices to know $M / 4$ points lying in the same quadrant to compute the rest of them. Each of these groups of $M / 4$ points of the same quadrant can be further divided into $I$ different energy circles according to their moduli. Moreover, in rectangular $M$ QAM constellations, the number $I$ of energies is, in general, ${ }^{9}$ smaller than the number of the points in a quadrant, $M / 4$. In other words, some energy circles have more than one point per quadrant.

Let $Q^{i}, 1 \leq i \leq I$, be the number of constellation points per quadrant that lie on the $i$ th energy circle. Thus, for the case of 16-QAM, we have $Q^{1}=1, Q^{2}=2$, and $Q^{3}=1$ points per energy quarter-circle, where $i=1,2,3$ refer, respectively, to the innermost, the middle, and the outermost circles (see Figure 4(a)). Furthermore, we denote by $x_{q}^{i}$ and $\theta_{q}^{i}$ each point of a quarter-circle and its phase, respectively, where $0 \leq q \leq Q^{i}-1$ is the point's index. Starting the numbering anticlockwise from the positive real axis, we may define the (relative) angle of the $q$ th point on the $i$ th energy level as

$$
\Delta \theta_{q}^{i}=\theta_{q}^{i}-\theta_{0}^{i}, \quad 0 \leq q \leq Q^{i}-1,1 \leq i \leq I,
$$

\footnotetext{
${ }^{9}$ Only for 4 -QAM, $I=M / 4$.
} 
where $\theta_{0}^{i}$ is the phase of the first point to meet, moving anticlockwise, on the ith energy circle.

As already noted, the relative angles $\Delta \theta_{q}^{i}$ are not affected by the nonlinearity and can therefore be assumed to be a priori known. Thus, once the value for the contribution $c_{x_{0}^{i}}^{m}$ of a channel tap corresponding to the symbol $x_{0}^{i}$ on the $i$ th level has been estimated, the remaining contribution values of that tap for symbols in the same quadrant and on the same energy level may be computed via rotations with predetermined constant angles as

$$
c_{x_{q}^{i}}^{m}=c_{x_{0}^{i}}^{m} \cdot e^{j \Delta \theta_{q}^{i}}, \quad 1 \leq q \leq Q^{i}-1,1 \leq i \leq I, 0 \leq m \leq L-1 .
$$

Once we have computed the subset of contribution values $c_{x_{q}^{i}}^{m}, 1 \leq i \leq I$, which correspond to the points of the first quadrant of the input constellation, estimates for the whole set of $c_{x}^{m}$ 's can be obtained by simple $\pi / 2$ rotations on the complex plane. This exploits the fact that the symbols in a quadrant are positioned at the corners of squares centered on the origin.

We can conclude that the estimation of only one contribution value per energy level and per channel tap is sufficient. With $L$ taps and $I$ energy levels, the number of contributions to be estimated directly from the training data amounts then to only $I \cdot L$, instead of $M \cdot L$. These contributions are computed with the aid of the estimates of the centers of $I \cdot L$ properly selected clusters in a manner analogous to that followed in the CBSE for the linear case.

Example $1(H(z)=9-9 j(L=1))$. Consider the example of a single-tap channel with 16-QAM input. The parameters of the nonlinearity model in (3), (4) are set to their typical values [21]. The input alphabet is

$$
\begin{aligned}
& S=\{1+j,-1+j,-1-j, 1-j, \\
& \\
& 3+j,-1+3 j,-3-j, 1-3 j, \\
& 1+3 j,-3+j,-1-3 j, 3-j, \\
&3+3 j,-3+3 j,-3-3 j, 3-3 j\} .
\end{aligned}
$$

Using the above notation, we will have $x_{0}^{1}=1+j, x_{0}^{2}=3+j$, $x_{1}^{2}=1+3 j, x_{0}^{3}=3+3 j$. Hence the above set can be written as

$$
\begin{array}{r}
S=\left\{x_{0}^{1}, j \cdot x_{0}^{1},-x_{0}^{1},-j \cdot x_{0}^{1},\right. \\
x_{0}^{2}, j \cdot x_{0}^{2},-x_{0}^{2},-j \cdot x_{0}^{2}, \\
x_{1}^{2}, j \cdot x_{1}^{2},-x_{1}^{2},-j \cdot x_{1}^{2}, \\
\left.x_{0}^{3}, j \cdot x_{0}^{3},-x_{0}^{3},-j \cdot x_{0}^{3}\right\},
\end{array}
$$

where all constellation points on the complex plane are depicted in Figure 4(a).

One can see that, before the application of the TWTA, we have 16 points grouped in 4 squares and $M / 4=4$ of these are located in the first quadrant of the signal space, distributed on $I=3$ energy levels. One point $\left(x_{0}^{1}\right)$ at the innermost level $(i=1)$, two $\left(x_{0}^{2}, x_{1}^{2}\right)$ at the middle level $(i=2)$, and one $\left(x_{0}^{3}\right)$ at the outermost level $(i=3)$. The angle $\Delta \theta_{1}^{2}$ between the two points of the middle energy level is defined by (14). At the output of the amplifier we still have three distinct energy levels (Figure 4(b)). It is easy to see that the original four squares retain also their structure after the action of the nonlinearity.

In this extreme case of a single-tap channel, the received observations form 16 different clusters on the complex plane, located at the corners of 4 different squares, whose size and angle depend on the single channel tap $h_{0}$ (see Figure 6(a)). Each one of the centers corresponds to one, among 16 possible transmitted symbols, $x$, as shown in Figure 6(b).

The 16 contributions, $c_{x}^{0}$, defined for the tap $h_{0}$, coincide, in this case, with the centers $\bar{y}$. Having estimated only the 3 contributions $c_{x_{0}^{1}}^{0}, c_{x_{0}^{2}}^{0}$, and $c_{x_{0}^{3}}^{0}$, we may compute the contribution $c_{x_{1}^{2}}^{0}$ with the aid of (15):

$$
c_{x_{1}^{2}}^{0}=c_{x_{0}^{2}}^{0} \cdot e^{j \Delta \theta_{1}^{2}}
$$

and then, via simple $\pi / 2$ rotations, all the remaining 12 tap contributions, $c_{x}^{0}$.

Example $2\left(H(z)=(9-9 j)+(1-0.1 j) z^{-1}(L=2)\right)$. In this example, a second tap, $1-0.1 j$, has been added to the 1-tap channel of the previous example. Now each one of the centers corresponds to one of the possible transmitted 2-symbol combinations $\left[x_{k}, x_{k-1}\right]$ and we obtain the structure of Figure 7. Due to the contributions of the second tap, $c_{x}^{1}$, the observed centers are now positioned in 16 groups of 16 points each. The points around which these 16 groups are centered on are determined by the contributions of the first tap, $c_{x}^{0}$, which are associated with the transmitted symbol $x_{k}$. In Figure 7 we illustrate the contribution $c_{1+j}^{0}$ and the 16 possible contributions $c_{x}^{1}$ associated with the transmitted symbol $x_{k-1}$.

As in the previous example, we have $x_{0}^{1}=1+j, x_{0}^{2}=3+j$, and $x_{0}^{3}=3+3 j$. In addition to the three contributions $c_{x_{0}^{1}}^{0}$, $c_{x_{0}^{2}}^{0}$, and $c_{x_{0}^{3}}^{0}$, three more contributions $c_{x_{0}^{1}}^{1}, c_{x_{0}^{2}}^{1}$, and $c_{x_{0}^{3}}^{1}$ are now required in order to compute the contributions $c_{x_{1}^{2}}^{0}$ and $c_{x_{1}^{2}}^{1}$ with the aid of (15) and then, via simple $\pi / 2$ rotations, all the 32 tap contributions, $c_{x}^{m}$.

\subsection{Construction of the training sequence}

In order to construct a suitable training sequence, we follow a procedure similar to the one presented in Section 3.

(1) $I$ sequences of $L$ symbols, $\mathbf{x}_{\text {basic }}^{i}=\left[x_{q_{0}}^{i}, x_{q_{1}}^{i}, \ldots, x_{q_{L-1}}^{i}\right]$, $1 \leq i \leq I$, one for each energy level, are defined. We call them basic subsequences. Not any $L$ QAM symbols are appropriate for such a sequence. We have to conform to the following constraints.

(a) The symbols must be located in the first quadrant of the signal space.

(b) They must lie on the same energy circle, $i$.

Thus, and in accordance with our choice for $\mathbf{x}_{\text {basic }}$ in the linear channel case, we choose $x_{q_{0}}^{i}=x_{q_{1}}^{i}=\cdots=x_{q_{L-1}}^{i}=x_{0}^{i}$. 


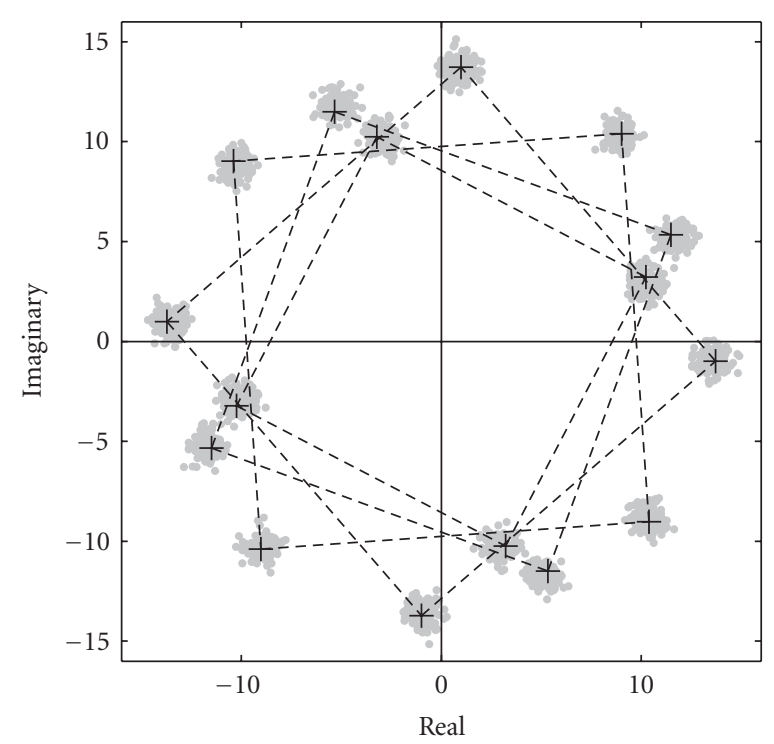

(a)

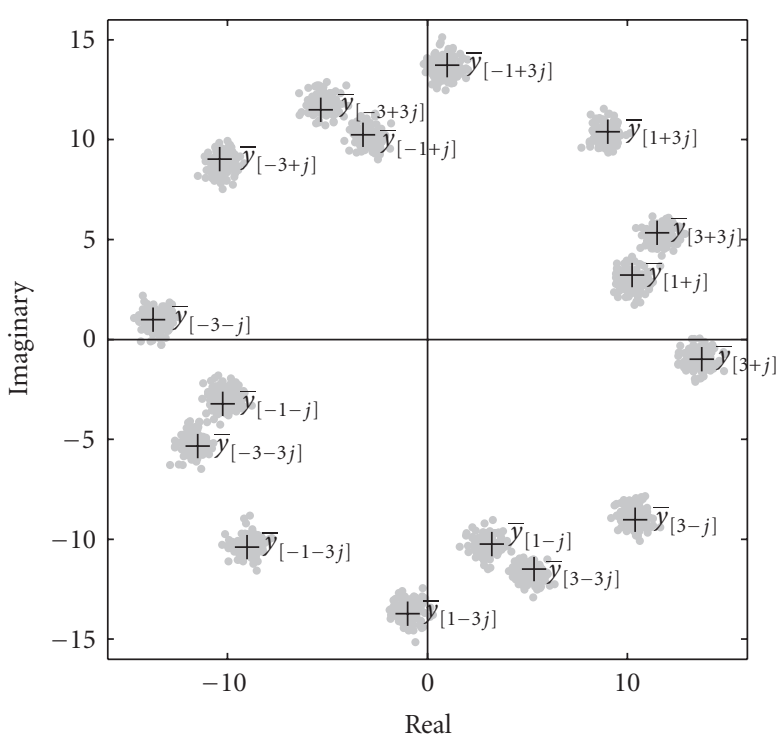

(b)

FIgURe 6: Plot of the clusters formed by a single-tap channel with 16-QAM input. (a) The formed squares and (b) the cluster centers associated with the corresponding transmitted symbols are shown. The crosses denote the cluster centers and the dots are the noise-corrupted observations for $20 \mathrm{~dB}$ SNR.

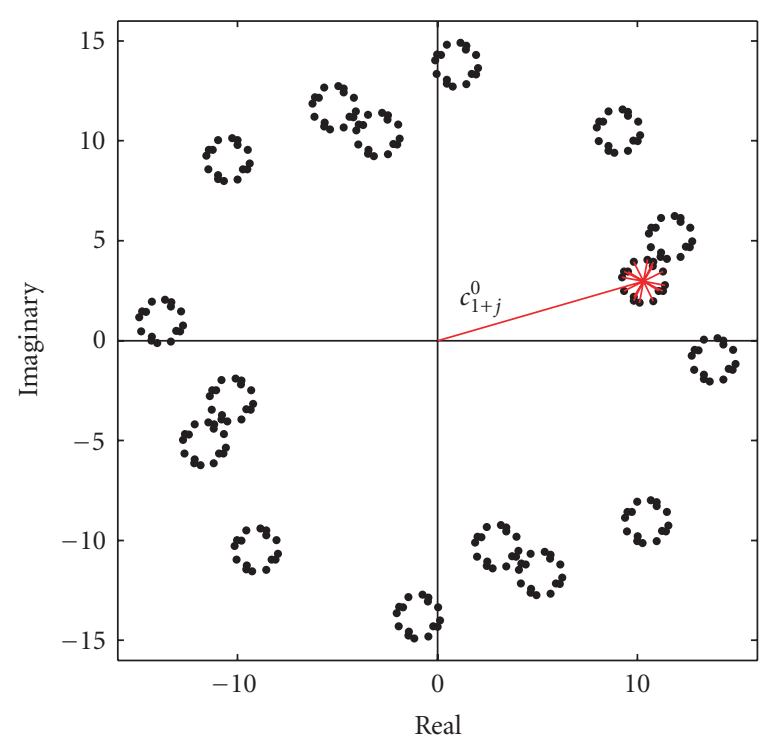

(a)

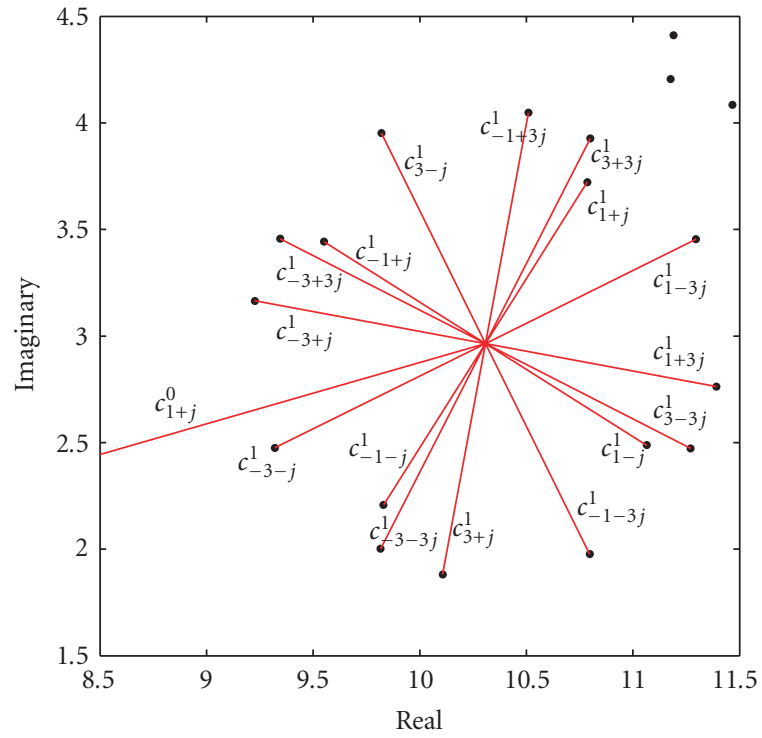

(b)

Figure 7: (a) Plot of the clusters formed when a 2-tap channel is used. The tap contribution $c_{1+j}^{0}$ as well as all tap contributions $c_{x}^{1}$ are shown in detail in (b).

The point $x_{0}^{i}$ is selected from each energy level $i$ of the input constellation so as to have minimum phase $\theta_{0}^{i}$ (following the notation in Section 4.2). The associated observed center is called the basic center, $C_{\text {basic }}^{i}=\bar{y}_{\left[x_{0}^{i}, x_{0}^{i}, \ldots, x_{0}^{i}\right]}$, of the $i$ th energy level. Each basic subsequence generates the $L$ centers $C_{m}^{i}$, $0 \leq m \leq L-1$, required for the computation of the channel tap contributions, $c_{x_{0}^{i}}^{m}$, as shown in Table 1.
(2) Define the subsequence, $\operatorname{subtr}^{i}$, as

$$
\operatorname{subtr}^{i} \triangleq[\underbrace{x_{0}^{i}, x_{0}^{i}, \ldots, x_{0}^{i}}_{L-1 \text { times }},-x_{0}^{i}],
$$

and let $\operatorname{tr}^{i}$ denote the periodic repetition of subtri ${ }^{i}$,

$$
\operatorname{tr}^{i} \triangleq\left[\operatorname{subtr}^{i}, \operatorname{subtr}^{i}, \ldots, \operatorname{subtr}^{i}\right] .
$$


TABLE 1: The $L$ cluster centers required for the estimation of the tap contributions for the $i$ th energy level.

\begin{tabular}{c|c}
\hline$C_{0}^{i}$ & $\bar{y}_{\left[-x_{0}^{i}, x_{0}^{i}, \ldots, x_{0}^{i}, \ldots, x_{0}^{i}\right]}$ \\
$C_{1}^{i}$ & $\bar{y}_{\left[x_{0}^{i},-x_{0}^{i}, \ldots, x_{0}^{i}, \ldots, x_{0}^{i}\right]}$ \\
$\vdots$ & $\vdots$ \\
$C_{m}^{i}$ & $\bar{y}_{\left[x_{0}^{i}, x_{0}^{i}, \ldots,-x_{0}^{i}, \ldots, x_{0}^{i}\right]}$ \\
$\vdots$ & $\vdots$ \\
$C_{L-1}^{i}$ & $\bar{y}_{\left[x_{0}^{i}, x_{0}^{i}, \ldots, x_{0}^{i}, \ldots,-x_{0}^{i}\right]}$ \\
\hline
\end{tabular}

We may then choose as the training sequence, tr, the following:

$$
\operatorname{tr}=\left[\operatorname{tr}^{1}, \operatorname{tr}^{2}, \ldots, \operatorname{tr}^{i}, \ldots, \operatorname{tr}^{I}\right]
$$

which generates observations ${ }^{10}$ for all the centers of Table 1. For $L=2, \operatorname{subtr}^{i} \triangleq\left[x_{0}^{i}, x_{0}^{i},-x_{0}^{i}\right] .^{11}$

\subsection{Summary of the proposed algorithm}

Once a training sequence has been constructed, the complete algorithm for the estimation of the transmitted symbols proceeds as follows.

Step 1. We estimate each of the $L \cdot I$ selected cluster centers by averaging the corresponding observations:

$$
C_{m}^{i}=\frac{1}{N^{(m, i)}} \sum_{k=1}^{N^{(m, i)}} y_{k}^{(m, i)}, \quad 0 \leq m \leq L-1,1 \leq i \leq I,
$$

where $y_{k}^{(m, i)}$ is the $k$ th observation for $C_{m}^{i}$ and $N^{(m, i)}$ is the number of observations associated with $C_{m}^{i}$. The basic center for the $i$ th level, $C_{\text {basic }}^{i}$, can then be computed based on the obtained estimates of the $L$ centers $C_{m}^{i}$ as follows:

$$
C_{\text {basic }}^{i}=\frac{\sum_{m=0}^{L-1} C_{m}^{i}}{L-2}, \quad L>2,1 \leq i \leq I .
$$

For $L \leq 2$, it turns out that we also have to estimate $C_{\text {basic }}^{i}$ directly from the training observations as in (22).

Step 2. The $I$ contributions, $c_{x_{0}^{i}}^{m}$, for each channel tap are computed as

$$
c_{x_{0}^{i}}^{m}=\frac{C_{\text {basic }}^{i}-C_{m}^{i}}{2}, \quad 0 \leq m \leq L-1,1 \leq i \leq I .
$$

\footnotetext{
${ }^{10}$ Note that the above training sequence gives rise to $L$-tuples containing mixed energy symbols as well. These are to be discarded in the training process.

11 Again, as explained in Section 3, slightly better performance could be obtained in the case of a two-path channel if the sequence $\left[x_{0}^{i}, x_{0}^{i}, x_{0}^{i},-x_{0}^{i}\right]$ was used instead.
}

Step 3. The $M / 4$ contributions for each channel tap that correspond to the points of the first quadrant are obtained with the aid of (15):

$$
c_{x_{q}^{i}}^{m}=c_{x_{0}^{i}}^{m} \cdot e^{j \Delta \theta_{q}^{i}}, \quad 1 \leq q \leq Q^{i}-1,1 \leq i \leq I, 0 \leq m \leq L-1,
$$

where the angles $\Delta \theta_{q}^{i}$ have been precalculated, and stored for each energy level, based on the knowledge of the signaling scheme, from (14).

Step 4. Via simple $n \cdot \pi / 2$ rotations, $1 \leq n \leq 3$, we obtain the rest of the $M$ contributions for each channel tap.

Step 5. All the remaining cluster centers $\bar{y}_{\left[x_{k}, x_{k-1}, \ldots, x_{k-L+1}\right]}$ are computed from (7).

Step 6. Finally, these centers are employed in the VA for the estimation of the transmitted symbol sequence.

Note that, for a single-tap channel $(L=1)$, the VA in Step 6 is reduced to a simple (nearest neighbor) decision step.

\section{Application of the algorithm to Example 2}

(i) We choose $x_{0}^{1}=1+j, x_{0}^{2}=3+j$, and $x_{0}^{3}=3+3 j$.

(ii) We compute $\Delta \theta_{1}^{2}$ for $x_{1}^{2}=1+3 j$ and $x_{0}^{2}=3+j$.

(iii) We choose the subsequences, subtr ${ }^{i}$, as

$$
\begin{gathered}
\operatorname{subtr}^{1} \triangleq[1+j, 1+j,-1-j], \\
\operatorname{subtr}^{2} \triangleq[3+j, 3+j,-3-j], \\
\operatorname{subtr}^{3} \triangleq[3+3 j, 3+3 j,-3-3 j]
\end{gathered}
$$

and periodically repeat them so as to have 10 training symbols per energy level:

$$
\begin{aligned}
\operatorname{tr}^{1} \triangleq & {[1+j, 1+j,-1-j, 1+j, 1+j,} \\
& -1-j, 1+j, 1+j,-1-j, 1+j], \\
\operatorname{tr}^{2} \triangleq & {[3+j, 3+j,-3-j, 3+j, 3+j,} \\
& -3-j, 3+j, 3+j,-3-j, 3+j], \\
\operatorname{tr}^{3} \triangleq[ & {[3+3 j, 3+3 j,-3-3 j, 3+3 j, 3+3 j,} \\
& -3-3 j, 3+3 j, 3+3 j,-3-3 j, 3+3 j] .
\end{aligned}
$$

The employed training sequence is

$$
\operatorname{tr}=\left[\operatorname{tr}^{1}, \operatorname{tr}^{2}, \operatorname{tr}^{3}\right]
$$

The resulting observations are used to estimate the $L \cdot I=6$ selected centers as in (22). These centers are depicted in Figure 8.

(iv) From (24) we obtain $c_{x_{0}^{1}}^{0}, c_{x_{0}^{2}}^{0}, c_{x_{0}^{3}}^{0}, c_{x_{0}^{1}}^{1}, c_{x_{0}^{2}}^{1}$, and $c_{x_{0}^{3}}^{1}$.

(v) From the above and (15) we obtain $c_{x_{1}^{2}}^{0}$ and $c_{x_{1}^{2}}^{1}$.

(vi) We estimate, via simple $\pi / 2$ rotations, all the rest of the tap contributions, $c_{x}^{m}$. 


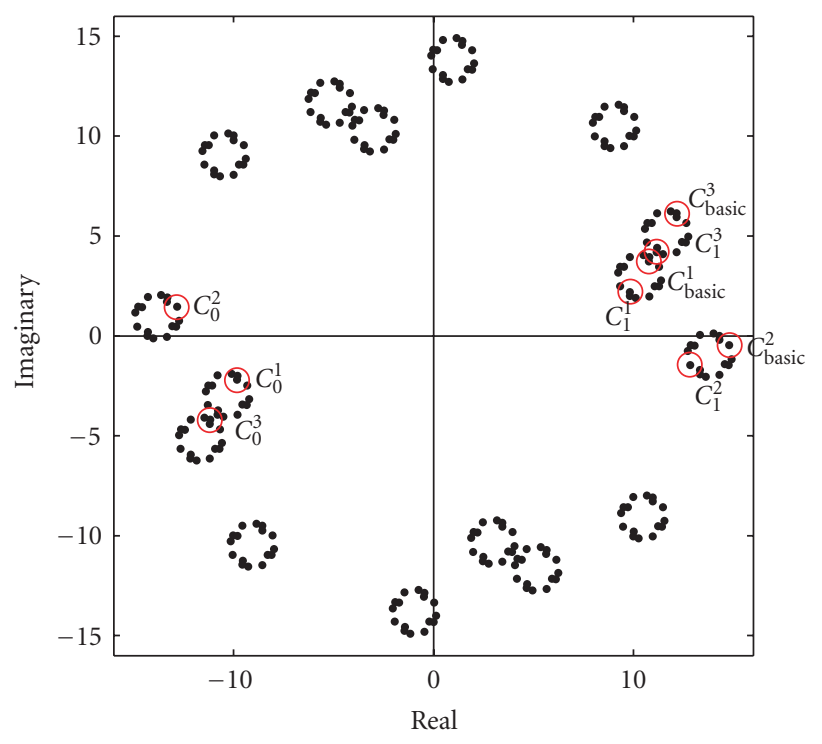

FIgURE 8: Cluster center constellation at the output of the TWTA for the channel of Example 2. The centers that are estimated directly from training data are denoted with circles.

(vii) We compute all cluster centers $\bar{y}_{\left[x_{k}, x_{k-1}\right]}$ from (7).

(viii) Finally, we use these centers in the VA to estimate the transmitted symbol sequence.

\section{A COMPARISON WITH OTHER EQUALIZERS}

In this section, the performance of the proposed equalizer is compared with a conventional linear transversal equalizer (LTE) and with two of the most widely used nonlinear equalizers: a Volterra series equalizer $[26,36,37]$ and an RBF equalizer $[38,39]$. The algorithms are compared in terms of the resulting bit error rates (BER) and their computational requirements.

Both 4-QAM and 16-QAM signaling schemes are considered. Two channel types are examined: an AWGN channel $(L=1)$ and a 2-tap $(L=2)$ stationary channel. The latter was chosen so that to simulate realistic conditions [5]. Its transfer function is $H(z)=(1-0.5 j)+(0.3+0.2 j) z^{-1}$, having a difference of $8 \mathrm{~dB}$ in magnitude between the first and the second taps. The parameters for the nonlinearity model in (3), (4) assume their typical values, namely, $\alpha_{a}=2.1587$, $\beta_{a}=1.1517, \alpha_{p}=4.0033$, and $\beta_{p}=9.104$ [21]. The input vectors for the LTE and Volterra equalizers are of length 3 . In these equalizers, the equalization delay was set to zero (since minimum-phase channels were used). The comparative performance results reported here are typical for a number of other channels used.

\subsection{Linear transversal equalizer}

For the LTE, a conventional adaptive linear filter, employing the normalized LMS (NLMS) algorithm [40], was used. The step-size, $\mu$, has been chosen so as to optimize the MSE for
TABLE 2: Experiment parameters for the LTE and Volterra equalizers (zero equalization delay).

\begin{tabular}{c|c|c|c|cr}
\hline & \multirow{2}{*}{$\begin{array}{l}\text { IBO } \\
(\mathrm{dB})\end{array}$} & $L$ & LTE & \multicolumn{2}{|c}{ Volterra } \\
\cline { 3 - 6 } 4-QAM & \multirow{2}{*}{0} & 1 & 0.1 & 0.6 & 2048 \\
& & 2 & 0.1 & 1.0 & 512 \\
\hline \multirow{3}{*}{ 16-QAM } & \multirow{2}{*}{0} & 1 & 0.1 & 0.7 & 64 \\
& & 2 & 0.1 & 1.0 & 256 \\
\cline { 2 - 6 } & \multirow{2}{*}{-3} & 1 & 0.1 & 0.7 & 32 \\
& & 2 & 0.2 & 1.2 & 256 \\
\cline { 2 - 6 } & \multirow{2}{*}{-6} & 1 & 0.1 & 0.6 & 32 \\
& & 2 & 0.2 & 1.2 & 256 \\
\hline
\end{tabular}

each particular case. The corresponding values are given in Table 2.

\subsection{Volterra equalizer}

The output of the Volterra equalizer used in the experiments is given by [37]

$$
\hat{x}_{n}=\sum_{i} q_{i} y_{n-i}+\sum_{i} \sum_{j} \sum_{k} q_{i, j, k} y_{n-i} y_{n-j} y_{n-k}^{*} .
$$

Thus, the output of the equalizer consists of a weighted linear and nonlinear combination of channel outputs, with complex weights. Weights $q_{i}$ multiply the channel outputs $y_{n}$ directly, and the weights $q_{i, j, k}$ multiply third-order products of the channel outputs. Only odd-order terms are considered, since even-order terms fall out of the frequency band of interest [26]. The order of the equalizer is restricted to three, because of the prohibitive increase in computational complexity as well as convergence time that higher-order terms would imply. The NLMS algorithm, with different step-sizes for the linear and the nonlinear parts [11], was used to adapt the Volterra weights. The parameters of the algorithm (first-order step-size $\mu_{1}$, third-order step-size $\mu_{3}$ ) have been chosen so as to optimize the MSE for each case and are given in Table 2. The third-order step-size is related to the first-order step-size as $\mu_{3}=\mu_{1} / \nu$.

\subsection{RBF-DF equalizer}

The performance of the proposed method is also compared with that of the symbol-by-symbol Bayesian decision feedback (DF) equalizer implemented via an RBF network $[38,39,41]$. A detailed description of the $M$-ary RBF-DF equalizer, considered here, can be found in [41]. Its structure is specified by the decision delay $\tau$, the feedforward order $n_{f}$ and the feedback order $n_{b}$. These parameters were chosen in relation to the length of the channel, $L$, as follows $[38,39,41]$ :

$$
\begin{gathered}
\tau=L-1, \quad n_{f}=\tau+1=L, \\
n_{b}=L+n_{f}-2-\tau=L-1 .
\end{gathered}
$$




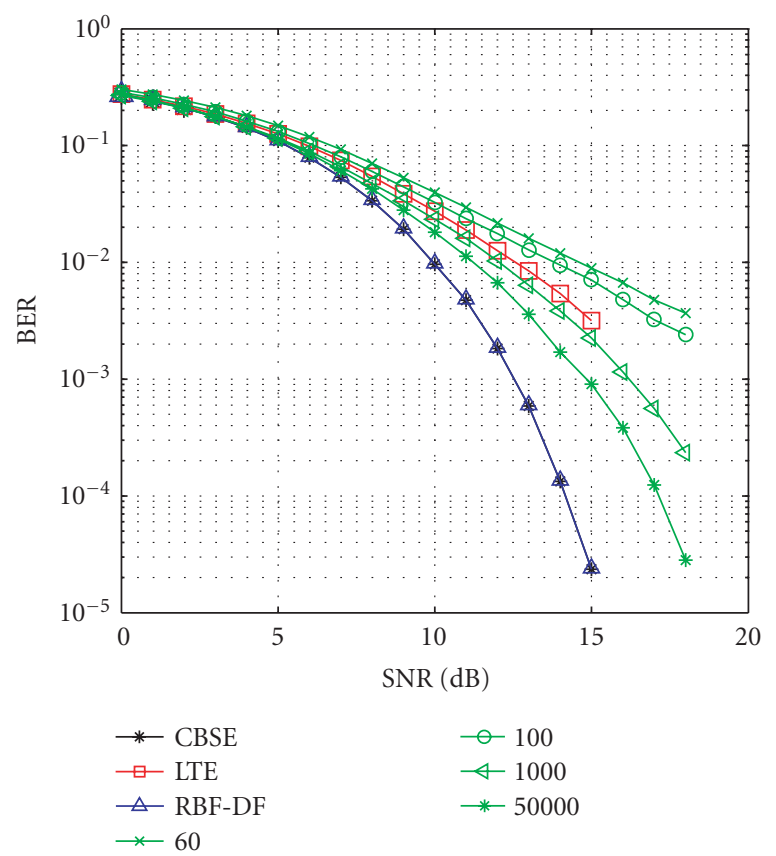

FIGURE 9: BER performance of the Volterra equalizer as a function of training sequence length, for an AWGN channel with 16-QAM input at $-6 \mathrm{~dB}$ IBO. $60,100,1000$, and 50000 training symbols per packet have been employed. The performance of the LTE (using 100 training symbols) and the CBSE and RBF-DF equalizers (with 60 training symbols) is also shown.

To make a fair comparison with the proposed CBSE algorithm, the centers computed by the latter were used to yield the centers for the $M$ sub-RBF networks of the RBF-DF equalizer. Moreover, the weights in the RBF networks were chosen in accordance with the assumption that all centers are equally probable.

\subsection{Simulation study}

The transmitted symbols are organized in packets consisting of two parts: the training symbols and 500 information symbols. The same training sequence is used for the CBSE and the RBF-DF equalizers. It consists of 20 specially selected symbols per energy zone, according to Section 4.3. Thus, in the case of 4-QAM (only one energy zone) the training sequence consists of 20 symbols whereas in the case of 16-QAM ( 3 energy zones) 3 times $20=60$ symbols are used for training. The number of samples was selected to be close to what is used in real systems such as GSM [42].

To determine the appropriate length for the training sequence of the Volterra equalizer, we have tried different cases but the tradeoff between computational complexity and performance gain led us to use only 100 randomly generated symbols in the comparison experiments. In Figure 9 a detailed performance comparison for the case of an AWGN channel and 16-QAM signaling at $-6 \mathrm{~dB}$ IBO with 60,100 , 1000 , and 50000 training symbols for the Volterra equalizer is given. For the LTE we also used 100 randomly generated
TABLE 3: Real operations required for cluster center estimation.

\begin{tabular}{lcc}
\hline Relation & Mul/Div & Add $/$ Sub \\
\hline Equation (22) & $2 I L$ & $2 I(N-L)$ \\
Equation (23) & $2 I$ & $2 I(L-1)$ \\
Equation (24) & $2 I L$ & $2 I L$ \\
Equation (15) & $4 L\left(\frac{M}{4}-I\right)$ & $2 L\left(\frac{M}{4}-I\right)$ \\
Equation (7) & 0 & $2(L-1)\left[\frac{M^{L}}{2}-2(L+1) I\right]$ \\
\hline & $M L+2 I$ & $2\left[I\left(N+1-2 L^{2}\right)\right.$ \\
Total & & $\left.+(L-1) \frac{M^{L}}{2}+L \frac{M}{4}\right]$ \\
\hline
\end{tabular}

symbols. For each equalizer, the BER is estimated once at least 100 symbol errors have been committed and at least 50 packets have been processed.

\subsection{1. $4-Q A M$}

The case of 4-QAM signaling with the TWT in saturation (at $0 \mathrm{~dB}$ IBO) was examined first. Due to the fact that the nonlinearity depends only on the signal amplitude, we may view the overall channel as linear in this case. The results are shown in Figure 10. One can see that for the AWGN channel $(L=1)$ all equalizers have roughly the same performance, whereas in the case of the second channel $(L=2)$ the CBSE and the Bayesian equalizers outperform the LTE and the Volterra equalizers.

\subsubsection{6-QAM}

For the 16-QAM signaling scheme three different cases for the nonlinearity were examined, where the IBO was set equal to $0 \mathrm{~dB},-3 \mathrm{~dB}$, and $-6 \mathrm{~dB}$ (Figures 11,12 , and 13). CBSE outperforms its competitors in every case. The Bayesian equalizer performs almost equally well, however, it is far more expensive in terms of computational requirements. It is of interest to note that even in the case of $0 \mathrm{~dB}$ IBO (full power efficiency [1]) with 16-QAM where other methods fail [5], the proposed equalizer still offers some gain. Notice also in Figure 9 the superior performance of CBSE over the Volterra equalizer trained with 50000 symbols.

\subsection{Computational requirements}

The overall computational load of the proposed equalizer consists of three parts, namely: (a) the computation of the tap contributions, (b) the computation of the cluster centers, and (c) the VA.

The cost of the first two parts, in terms of real multiplications and additions, is given analytically in Table 3 for $L>2$, where $N$ is the number of training samples per energy 
TABLE 4: Real operations required by each equalizer in the training part. $L_{\mathrm{LTE}}$ is the input vector length for the LTE. $L_{V}$ and $M_{V}$ are given by (31) and (32), respectively.

\begin{tabular}{|c|c|c|c|}
\hline Method & Mul/Div & Add/Sub & $(\cdot)^{2}$ \\
\hline $\begin{array}{l}\text { CBSE } \\
\text { RBF-DF }\end{array}$ & $M L+2 I$ & $2\left[I\left(N+1-2 L^{2}\right)+(L-1) \frac{M^{L}}{2}+L \frac{M}{4}\right]$ & 一 \\
\hline LTE & $N I\left(8 L_{\mathrm{LTE}}+3\right)$ & $10 N I L_{\mathrm{LTE}}$ & $2 N I L_{\mathrm{LTE}}$ \\
\hline Volterra & $N I\left[4 M_{V}+8 L_{V}+\frac{3}{2}(p+1)\right]$ & $N I\left(2 M_{V}+10 L_{V}\right)$ & $2 N I L_{V}$ \\
\hline
\end{tabular}

level. It is important to note that the number of multiplications/divisions required by the proposed method is independent of the amount of training data [22]. Divisions are performed once per training block. The operations counts given in this table hold for $L>2$. For $L=2$, these figures become $2 M$ multiplications/divisions and $2 I(N-6)+M(M+1)$ additions/subtractions. For $L=1$, we have $M-2 I$ multiplications/divisions and $2 I(N-2)+M / 2$ additions/subtractions.

Table 4 presents the computational cost for the training part in each equalizer. $L_{\mathrm{LTE}}$ is the length of the weight vector of the LTE equalizer. Assuming $L_{\mathrm{LTE}}-1$ delay elements for the $p$ th-order Volterra equalizer as well, it turns out that its corresponding weight vector is of length (recall that evenorder terms are not included):

$$
L_{V}=\sum_{n=0}^{(p-1) / 2}\left(\begin{array}{c}
n+L_{\mathrm{LTE}} \\
L_{\mathrm{LTE}}-1
\end{array}\right)\left(\begin{array}{c}
n+L_{\mathrm{LTE}}-1 \\
L_{\mathrm{LTE}}-1
\end{array}\right)
$$

In addition to the operations in the NLMS recursion, another

$$
M_{V}=\sum_{n=0}^{(p-1) / 2} 2 n\left(\begin{array}{c}
n+L_{\mathrm{LTE}} \\
L_{\mathrm{LTE}}-1
\end{array}\right)\left(\begin{array}{c}
n+L_{\mathrm{LTE}}-1 \\
L_{\mathrm{LTE}}-1
\end{array}\right)
$$

complex multiplications are required in the Volterra equalizer to form the regressor vector. ${ }^{12}$

The computational complexity per detected symbol of the VA part in the proposed method (part (c)), along with the corresponding complexities of the LTE, the Volterra, and the RBF-DF equalizers considered here are shown in Table 5.

Finally, Table 6 shows the total number of real operations required for the processing of a received block consisting of 20 training samples (per energy zone) and 500 data symbols, for a two-tap channel $(L=2)$ with the 16-QAM signaling scheme. For the purposes of this comparison, 3 times $20=60$ training samples are also assumed for the LTE and the Volterra equalizers, although, as we have already seen, this is not realistic and in practice a much longer data set is needed for these methods. For these equalizers,

12 In fact, this figure corresponds to a straightforward computation, where care is not taken to compute only once partial products that appear in several entries of the regressor vector. Nevertheless, such savings would not be significant with respect to the overall computational cost of the Volterra equalizer.
TABLE 5: Real operations required per detected symbol in the tested equalizers. $L_{L T E}$ is the input vector length for the LTE. $L_{V}$ and $M_{V}$ are given by (31) and (32), respectively.

\begin{tabular}{lcccc}
\hline Method & Mul/Div & Add/Sub & $(\cdot)^{2}$ & $\exp (\cdot)$ \\
\hline CBSE (VA) & 0 & $4 M^{L}$ & $2 M^{L}$ & - \\
RBF-DF & $M^{L}$ & $4 L M^{L}-M$ & $2 L M^{L}$ & $M^{L}$ \\
LTE & $4 L_{\mathrm{LTE}}$ & $4 L_{\mathrm{LTE}}-2$ & - & - \\
Volterra & $4\left(M_{V}+L_{V}\right)$ & $2\left(2 L_{V}+M_{V}-1\right)$ & - & - \\
\hline
\end{tabular}

we have $L_{\mathrm{LTE}}=3$ and $L_{V}=21$, respectively. Observe that the superior performance of the CBSE equalizer is attained at a substantially lower complexity, especially in terms of real multiplications/divisions.

\section{CONCLUSIONS}

A cluster-based sequence equalizer for satellite channels has been proposed for the case of rectangular QAM signaling. The method exploits the fact that TWT memoryless nonlinearities respect the symmetries underlying the signaling scheme, thus leading to a significant gain in performance, compared to Volterra and NN-based techniques, and at a significantly lower computational cost.

\section{APPENDIX}

Consider two points, $x_{0}^{i}, x_{1}^{i}$, of the input $M$-QAM constellation (located in the same quadrant-see Figure 5 for an example). The superscript $i$ indicates that they belong to the same (ith) energy level, $A^{i}$, of the constellation. Thus,

$$
x_{0}^{i}=u_{0}^{i}+j v_{0}^{i}, \quad x_{1}^{i}=u_{1}^{i}+j v_{1}^{i}
$$

with their magnitudes and phases being given by $A^{i}=$ $\sqrt{\left(u_{0}^{i}\right)^{2}+\left(v_{0}^{i}\right)^{2}}=\sqrt{\left(u_{1}^{i}\right)^{2}+\left(v_{1}^{i}\right)^{2}}$ and $\theta_{0}^{i}=\tan ^{-1}\left(v_{0}^{i} / u_{0}^{i}\right), \theta_{1}^{i}=$ $\tan ^{-1}\left(v_{1}^{i} / u_{1}^{i}\right)$, respectively. Then the angle between them is

$$
\Delta \theta_{1}^{i}=\theta_{1}^{i}-\theta_{0}^{i}
$$

Assume now that these two points do not belong to the same square (one cannot result from the other by simple $n \cdot \pi / 2$ 
TABLE 6: The total number of real operations for each equalizer, for a 2-tap channel $(L=2)$ with 16-QAM input, needed to process a packet of 60 training and 500 information samples; $L_{\mathrm{LTE}}=3, p=3, L_{V}=21, M_{V}=36$.

\begin{tabular}{|c|c|c|c|c|c|c|c|}
\hline \multirow[t]{2}{*}{ Method } & \multicolumn{2}{|c|}{ Mul/Div } & \multicolumn{2}{|c|}{ Add/Sub } & \multicolumn{2}{|c|}{$(\cdot)^{2}$} & \multirow{2}{*}{$\begin{array}{c}\exp (\cdot) \\
\text { Decision }\end{array}$} \\
\hline & Training & Decision & Training & Decision & Training & Decision & \\
\hline $\begin{array}{l}\text { CBSE } \\
\text { RBF-DF }\end{array}$ & 32 & $\begin{array}{c}0 \\
128000 \\
\end{array}$ & 356 & $\begin{array}{c}512000 \\
1016000 \\
\end{array}$ & - & $\begin{array}{l}256000 \\
512000 \\
\end{array}$ & $\begin{array}{c}- \\
128000\end{array}$ \\
\hline LTE & 1620 & 6000 & 1800 & 5000 & 360 & - & - \\
\hline Volterra & 19080 & 114000 & 16920 & 77000 & 2520 & - & - \\
\hline
\end{tabular}

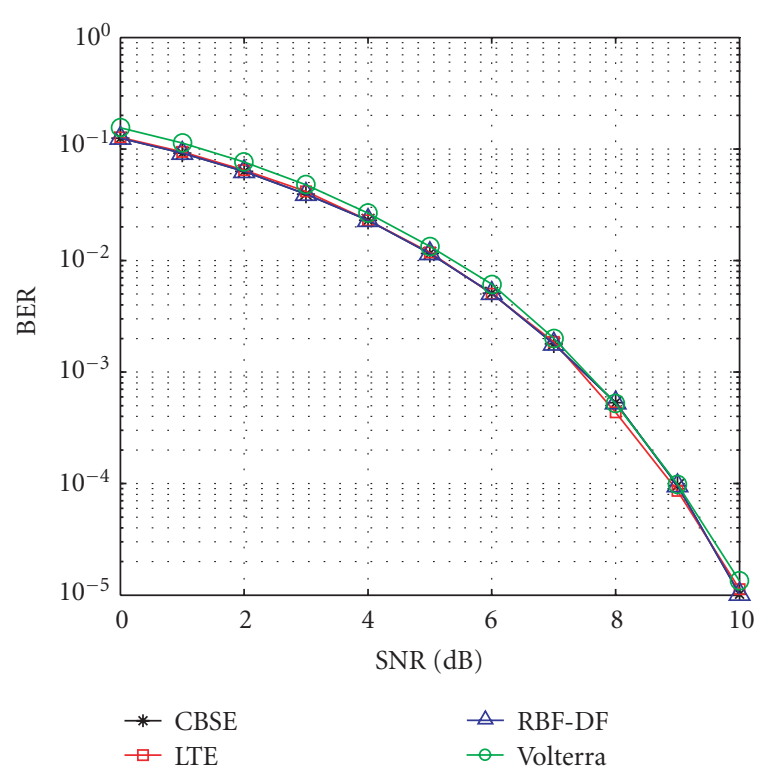

(a)

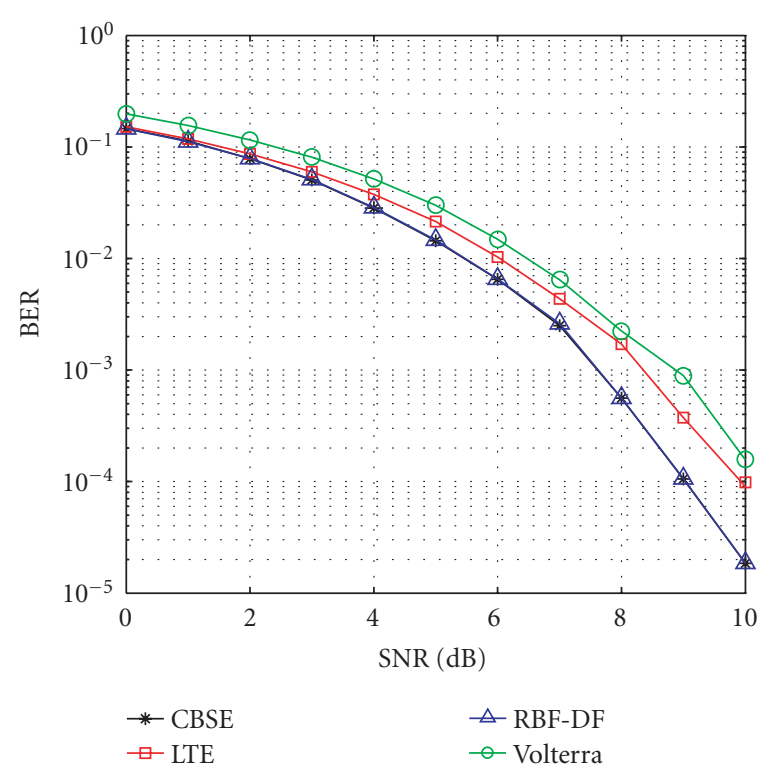

(b)

Figure 10: BER performance for 4-QAM at $0 \mathrm{~dB}$ IBO. (a) AWGN and (b) 2-tap channel.

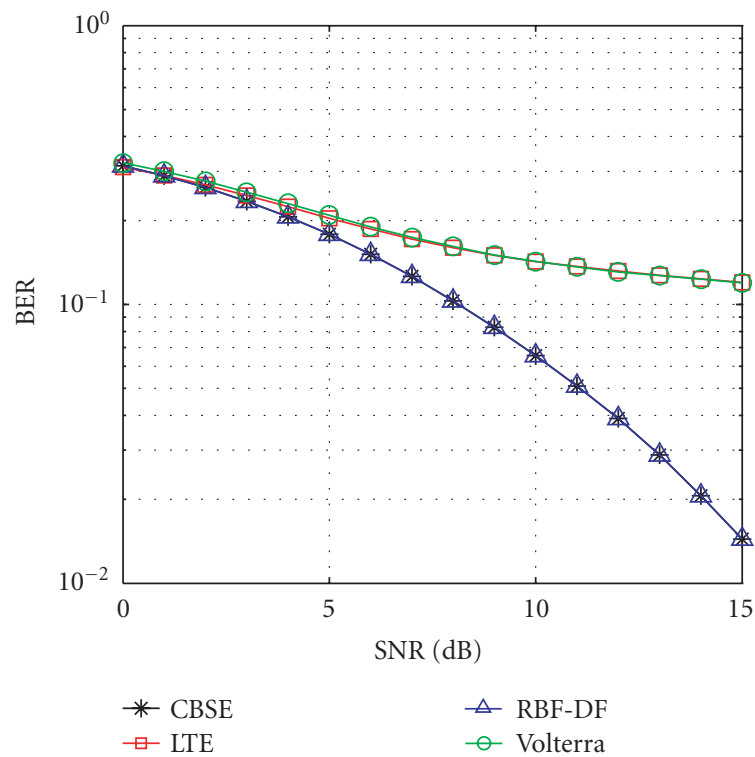

(a)

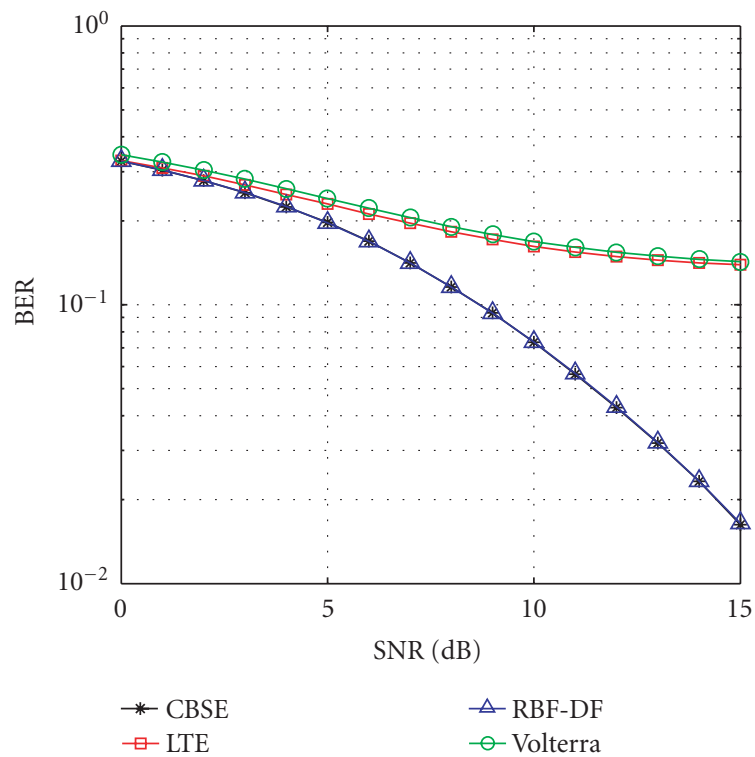

(b)

FIGURE 11: BER performance for 16-QAM at $0 \mathrm{~dB}$ IBO. (a) AWGN and (b) 2-tap channel. 


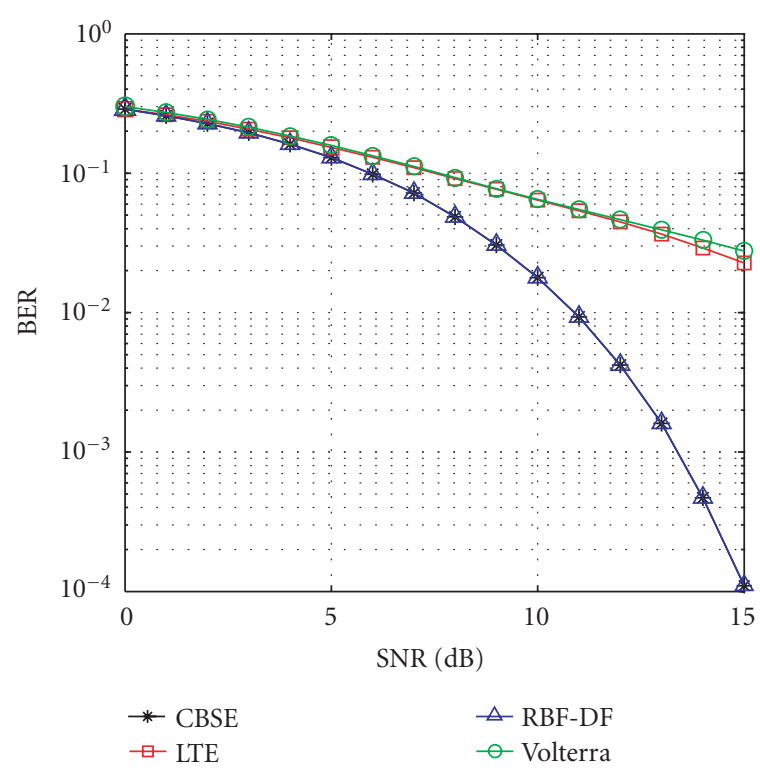

(a)

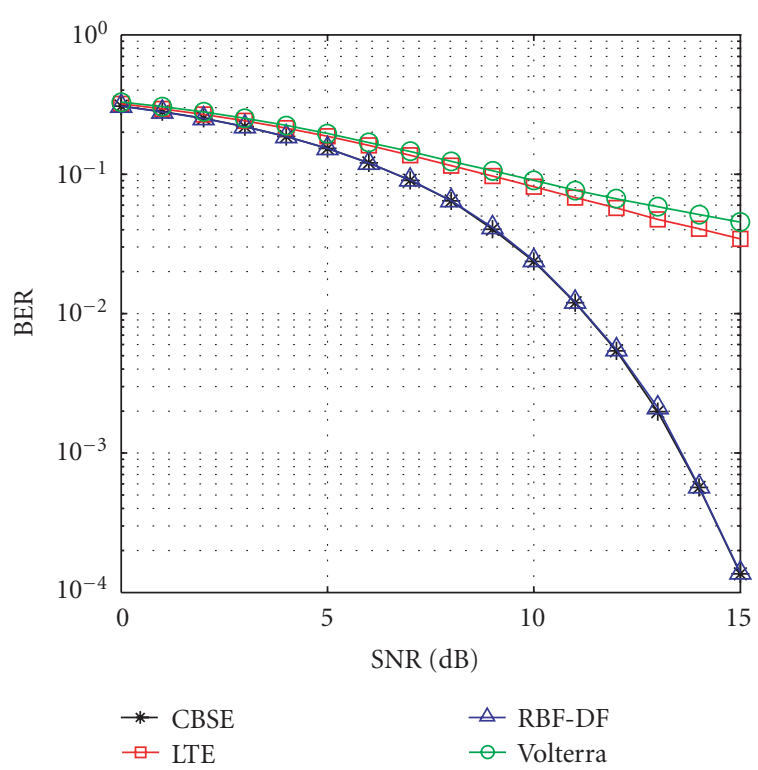

(b)

FIGURE 12: BER performance for 16-QAM at -3 dB IBO. (a) AWGN and (b) 2-tap channel.

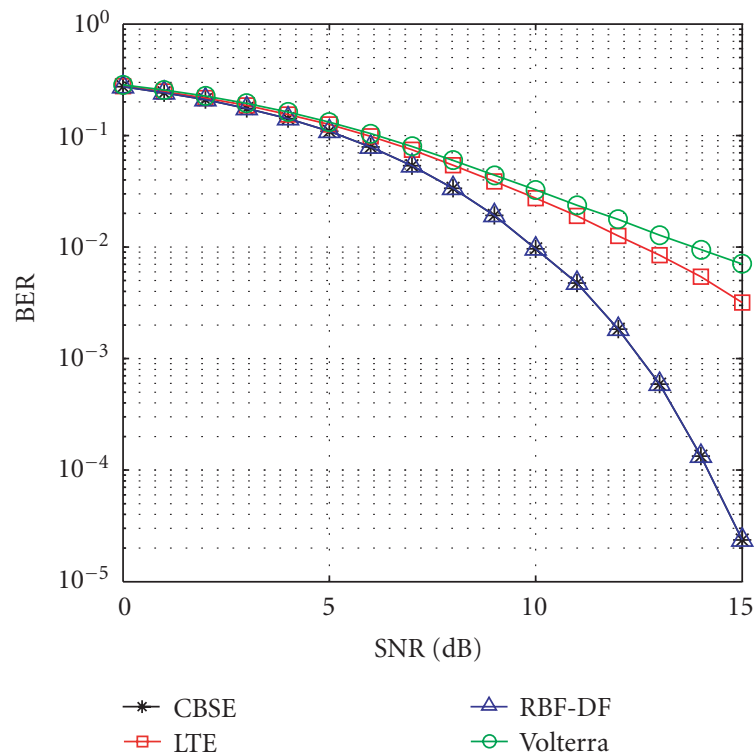

(a)

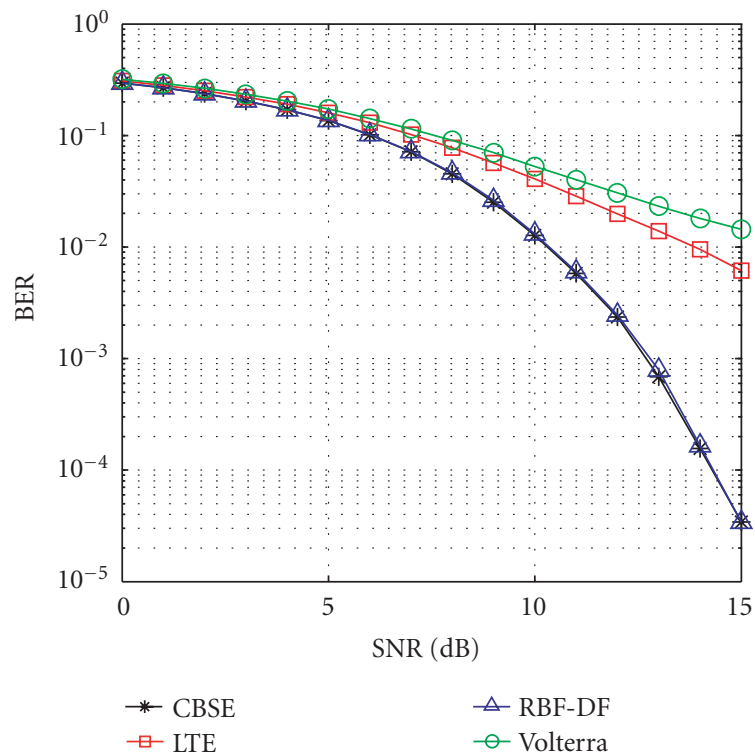

(b)

Figure 13: BER performance for 16-QAM at -6 dB IBO. (a) AWGN and (b) 2-tap channel.

rotations, where $1 \leq n \leq 3)$. Let $z_{0}^{i}, z_{1}^{i}$ be the corresponding outputs of the amplifier. Then

$$
\begin{aligned}
& z_{0}^{i}=x_{0}^{i} \cdot G\left(\left|x_{0}^{i}\right|\right)=g\left(A^{i}\right) e^{j\left[\theta_{0}^{i}+\Phi\left(A^{i}\right)\right]}, \\
& z_{1}^{i}=x_{1}^{i} \cdot G\left(\left|x_{1}^{i}\right|\right)=g\left(A^{i}\right) e^{j\left[\theta_{1}^{i}+\Phi\left(A^{i}\right)\right]}
\end{aligned}
$$

and the angle between them is given by

$$
\Delta \Theta=\left[\theta_{1}^{i}+\Phi\left(A^{i}\right)\right]-\left[\theta_{0}^{i}+\Phi\left(A^{i}\right)\right]=\theta_{1}^{i}-\theta_{0}^{i}=\Delta \theta_{1}^{i}
$$

that is, it is not affected by the nonlinearity. 


\section{ACKNOWLEDGMENT}

Fruitful discussions with Dr. Daniel Roviras, TeSA/IRIT, Toulouse, France, are gratefully acknowledged.

\section{REFERENCES}

[1] G. Maral and M. Bousquet, Satellite Communication Systems, John Wiley \& Sons, New York, NY, USA, 1996.

[2] M. Ibnkahla, Q. M. Rahman, A. I. Sulyman, H. A. Al-Asady, J. Yuan, and A. Safwat, "High-speed satellite mobile communications: technologies and challenges," Proceedings of the IEEE, vol. 92, no. 2, pp. 312-338, 2004.

[3] F. Xiong, "Modem techniques in satellite communications," IEEE Communications Magazine, vol. 32, no. 8, pp. 84-98, 1994.

[4] E. Biglieri, A. Gersho, R. D. Gitlin, and T. L. Lim, "Adaptive cancellation of nonlinear intersymbol interference for voiceband data transmission," IEEE Journal on Selected Areas in Communications, vol. 2, no. 5, pp. 765-777, 1984.

[5] S. Bouchired, D. Roviras, and F. Castanié, "Equalization of satellite mobile channels with neural network techniques," Space Communications, vol. 15, no. 4, pp. 209-220, 1999.

[6] F. Langlet, H. Abdulkader, D. Roviras, A. Mallet, and F. Castanié, "Comparison of neural network adaptive predistortion techniques for satellite down links," in Proceedings of the International Joint Conference on Neural Networks (IJCNN '01), vol. 1, pp. 709-714, Washington, DC, USA, July 2001.

[7] F. Langlet, D. Roviras, A. Mallet, and F. Castanié, "Mixed analog/digital implementation of MLP NN for predistortion," in Proceedings of the International Joint Conference on Neural Networks (IJCNN '02), vol. 3, pp. 2825-2830, Honolulu, Hawaii, USA, May 2002.

[8] F. Langlet, H. Abdulkader, and D. Roviras, "Predistortion of non-linear satellite channels using neural networks: architecture, algorithm and implementation," in Proceedings of the 11th European Signal Processing Conference (EUSIPCO '02), Toulouse, France, September 2002.

[9] S. Haykin, Neural Networks: A Comprehensive Foundation, Prentice-Hall, Upper Saddle River, NJ, USA, 2nd edition, 1999.

[10] A. Gutierrez and W. E. Ryan, "Performance of adaptive Volterra equalizers on nonlinear satellite channels," in IEEE International Conference on Communications (ICC '95), vol. 1, pp. 488-492, Seattle, Wash, USA, June 1995.

[11] A. Gutierrez and W. E. Ryan, "Performance of Volterra and MLSD receivers for nonlinear band-limited satellite systems," IEEE Transactions on Communications, vol. 48, no. 7, pp. 1171$1177,2000$.

[12] S. Benedetto, E. Biglieri, and R. Daffara, "Modeling and performance evaluation of nonlinear satellite links-a Volterra series approach," IEEE Transactions on Aerospace and Electronic Systems, vol. 15, no. 4, pp. 494-507, 1979.

[13] P.-R. Chang and B.-C. Wang, "Adaptive decision feedback equalization for digital satellite channels using multilayer neural networks," IEEE Journal on Selected Areas in Communications, vol. 13, no. 2, pp. 316-324, 1995.

[14] S. Chen, G. J. Gibson, C. F. N. Cowan, and P. M. Grant, "Adaptive equalization of finite non-linear channels using multilayer perceptrons," Signal Processing, vol. 20, no. 2, pp. 107-119, 1990.

[15] I. Cha and S. A. Kassam, "Channel equalization using adaptive complex radial basis function networks," IEEE Journal on Selected Areas in Communications, vol. 13, no. 1, pp. 122-131, 1995.
[16] S. Chen, S. McLaughlin, and B. Mulgrew, "Complex-valued radial basis function network, part 1: network architecture and learning algorithms," Signal Processing, vol. 35, no. 1, pp. 1931, 1994.

[17] S. Bouchired, M. Ibnkahla, and W. Paquier, "A combined LMS-SOM algorithm for time varying non-linear channel equalization," in Proceedings of the European Signal Processing Conference (EUSIPCO '98), Rhodes, Greece, September 1998.

[18] S. Bouchired, M. Ibnkahla, D. Roviras, and F. Castanié, "Equalization of satellite mobile communication channels using combined self-organizing maps and RBF networks," in Proceedings of the International Conference on Acoustics, Speech, and Signal Processing (ICASSP '98), vol. 6, pp. 33773379, Seattle, Wash, USA, May 1998.

[19] T. Kohonen, Self-Organizing Maps, Springer, Berlin, Germany, 1995.

[20] S. Bouchired, M. Ibnkahla, D. Roviras, and F. Castanié, "Equalization of satellite UMTS channels using neural network devices," in Proceedings of the IEEE International Conference on Acoustics, Speech and Signal Processing (ICASSP '99), Phoenix, Ariz, USA, March 1999.

[21] A. A. M. Saleh, "Frequency-independent and frequency dependent nonlinear models of TWT amplifiers," IEEE Transactions on Communications, vol. 29, no. 11, pp. 1715-1720, 1981.

[22] Y. Kopsinis and S. Theodoridis, "An efficient low-complexity technique for MLSE equalizers for linear and nonlinear channels," IEEE Transactions on Signal Processing, vol. 51, no. 12, pp. 3236-3248, 2003.

[23] Y. Kopsinis and S. Theodoridis, "A novel cluster based MLSE equalizer for $M$-PAM signaling schemes," Signal Processing, vol. 83, no. 9, pp. 1905-1918, 2003.

[24] G. D. Forney Jr, "Maximum-likelihood sequence estimation of digital sequences in the presence of intersymbol interference," IEEE Transactions on Information Theory, vol. 18, no. 3, pp. 363-378, 1972.

[25] D. Chakraborty, "Maximum likelihood sequence detection in nonlinear satellite channels," IEEE Communications Magazine, vol. 19 , no. 6, pp. 47-53, 1981.

[26] S. Benedetto, E. Biglieri, and V. Castellani, Digital Transmission Theory, Prentice-Hall, Englewood Cliffs, NJ, USA, 1987.

[27] P. Lowry, "System handbook, advanced communications technology satellite," Tech. Rep. TM-101490, NASA, Lewis Research Center, Cleveland, Ohio, USA, 1993, http://acts.grc. nasa.gov/docs/ACTSSysHB.PDF.

[28] ESA SkyPlex, http://telecom.esa.int/telecom/www/object/index.cfm? fobjectid=12143.

[29] M. Wittig, "Satellite onboard processing for multimedia applications," IEEE Communications Magazine, vol. 38, no. 6, pp. 134-140, 2000.

[30] B. Evans, M. Werner, E. Lutz, et al., "Integration of satellite and terrestrial systems in future multimedia communications," IEEE Wireless Communications, vol. 12, no. 5, pp. 7280, 2005.

[31] M. Ibnkahla and J. Yuan, "A neural network MLSE receiver based on natural gradient descent: application to satellite communications," EURASIP Journal on Applied Signal Processing, vol. 2004, no. 16, pp. 2580-2591, 2004.

[32] C. Rapp, "Effects of HPA-nonlinearity on a 4-DPSK/OFDMsignal for a digital sound broadcasting system," in Proceedings of the 2nd European Conference on Satellite Communications (ECSC '91), Liege, Belgium, October 1991.

[33] J. M. Weekley and B. J. Mangus, "TWTA versus SSPA: A comparison of on-orbit reliability data," IEEE Transactions on Electron Devices, vol. 52, no. 5, pp. 650-652, May 2005. 
[34] A. L. Berman and C. E. Mahle, "Nonlinear phase shift in travelling wave as applied to multiple access communication satellites," IEEE Transactions on Communications Technology, vol. 198, no. 1, pp. 37-48, 1970.

[35] J. B. Minkoff, "Wideband operation of nonlinear solid state power amplifiers-comparison of calculations and measurements," AT\&T Bell Laboratories Technical Journal, vol. 63, no. 2, pp. 231-248, 1984.

[36] V. J. Mathews, “Adaptive polynomial filters," IEEE Signal Processing Magazine, vol. 8, no. 3, pp. 10-26, 1991.

[37] S. Benedetto and E. Biglieri, "Nonlinear equalization of digital satellite channels," IEEE Journal on Selected Areas in Communications, vol. 1, no. 1, pp. 57-62, 1983.

[38] S. Chen, B. Mulgrew, and S. McLaughlin, "Adaptive Bayesian equalizer with decision feedback," IEEE Transactions on Signal Processing, vol. 41, no. 9, pp. 2918-2926, 1993.

[39] S. Chen, S. McLaughlin, and B. Mulgrew, "Complex-valued radial basis function network, part II: application to digital communications channel equalisation," Signal Processing, vol. 36, no. 2, pp. 175-188, 1994.

[40] S. Haykin, Adaptive Filter Theory, Prentice-Hall, Upper Saddle River, NJ, USA, 3rd edition, 1996.

[41] L. Hanzo, C. H. Wong, and M. S. Yee, Adaptive Wireless Transceivers, John Wiley \& Sons, Chichester, UK, 2002.

[42] R. Steele and L. Hanzo, Mobile Radio Communications, John Wiley \& Sons, Chichester, UK, 2nd edition, 2000.

Eleftherios Kofidis was born on September 6, 1967, in Trikala, Greece. He received the diploma (with honors) and the Ph.D. degrees, in 1990 and 1996, respectively, both from the Department of Computer Engineering and Informatics, University of Patras, Patras, Greece. From 1996 to 1998 he was with the Greek Army. From 1998 to 2000 he was a postdoctoral research fellow with the Communications, Image and Information Processing Department, Institut National des Télécommunications, Evry, France. From 2001 to 2004 he held adjunct professor positions with the universities of Piraeus and Peloponnese, Greece. He is currently a Lecturer of informatics in the Department of Statistics and Insurance Science, University of Piraeus, Piraeus, Greece. His current research interests are in signal processing for communications. He is a member of the Technical Chamber of Greece.

Vassilis Dalakas was born in 1973, in Athens, Greece. He received his B.S. degree in Physics, in 1998, from the Department of Physics, University of Athens, Greece. From 1998 to 2000 he was with the Greek Army. He received the M.S. degree (honors.) in digital signal processing from the Department of Informatics and Telecommunications, University of Athens, in 2002. He is currently working towards a Ph.D. in signal processing at the same institution, in cooperation with the Institute for Space Applications and Remote Sensing of the National Observatory of Athens. Since October 2001, he has been a Research Fellow with the Department of Geography, Harokopio University of Athens. His research interests include signal processing for communications systems and modeling and simulation standardization methods.
Yannis Kopsinis was born in Patra, Greece, on May 1, 1973. He received the B.S. degree in informatics from the Department of Informatics and Telecommunications, University of Athens, Greece, in 1998 and his Ph.D. degree in 2003 from the same department. Since January 2004 he has been a research fellow with the Institute for Digital Communications, School of Engineering and Electronics, the University of Ed-

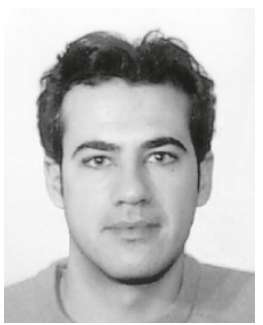
inburgh, UK. His research interests include channel equalization, adaptive filters, and electronic signal processing and performance evaluation for optical communication systems.

Sergios Theodoridis received an Honors degree in physics from the University of Athens and his M.S. and Ph.D. degrees from the Department of Electronics and Electrical Engineering of Birmingham University, UK. He is currently Professor of signal processing and communications in the Department of Informatics and Telecommunications of the University of Athens. His research interests lie in the areas of adaptive

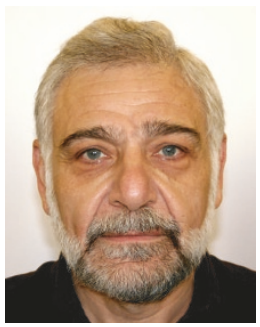
algorithms, channel equalization, pattern recognition, and signal processing for music. He is the Coeditor of the book Adaptive System Identification and Signal Processing Algorithms, Prentice Hall 1993, the Coauthor of the book Pattern Recognition, Academic Press, 3rd edition 2005, and the Coauthor of three books in Greek, two of them for the Greek Open University. He is currently an Associate Editor for the IEEE Signal Processing Magazine and he is a member of the editorial boards of the EURASIP Signal Processing Journal and EURASIP Journal on Wireless Communications and Networking. He was the general Chairman of EUSIPCO-98 and he is the Technical Program Cochair for ISCAS-2006. He is currently the President of EURASIP. 